\title{
Similarity of the concentration field of gas-phase turbulent jets
}

\author{
By DAVID R. DOWLING † AND PAUL E. DIMOTAKIS \\ Graduate Aeronautical Laboratories, California Institute of Technology, \\ Pasadena, CA 91125, USA
}

(Received 1 June 1989 and in revised form 9 December 1989)

This work is an experimental investigation of the turbulent concentration field formed when the nozzle gas from a round, momentum-driven, free turbulent jet mixes with gas entrained from a quiescent reservoir. The measurements, which were made with a non-intrusive laser-Rayleigh scattering diagnostic at Reynolds numbers of 5000,16000 , and 40000 , cover the axial range from 20 to 90 jet exit diameters and resolve the full range of temporal and spatial concentration scales. Reynoldsnumber-independent and Reynolds-number-dependent similarities are investigated. The mean and r.m.s. values of the concentration are found to be consistent with jet similarity laws. Concentration fluctuation power spectra are found to be self-similar along rays emanating from the virtual origin of the jet. The probability density function for the concentration is also found to be self-similar along rays. Near the centreline of the jet, the scaled probability density function of jet fluid concentration is found to be nearly independent of the Reynolds number.

\section{Introduction}

The round, momentum-driven, free turbulent jet, a small source of high-speed fluid issuing into a large quiescent reservoir, is one of the classical free shear flows. This simple geometry has wide engineering application and has attracted investigators for more than 50 years (Ruden 1933; Kuethe 1935). It is a special turbulent flow for several reasons, and two of its unique far-field properties are important elements of this investigation. First, the mean concentration field of a passive scalar that issues from the jet nozzle displays a distinct self-similarity with increasing downstream distance, and second, the evolution of the maximum mean velocity and the largest scale of motion conspire to give the flow a single Reynolds number independent of the distance from the jet nozzle (e.g. Landau \& Lifshitz 1959). Properties of the convected concentration (scalar) field, formed downstream of the jet nozzle, should depend only on the Reynolds number of the jet, the Schmidt number (kinematic viscosity divided by species diffusivity) of the jet/reservoir fluid pair, and the location of the observation point with respect to the nozzle exit. This paper explores these dependences by describing the results of an experimental investigation in gas-phase turbulent jets, and comparing the findings with previous jet studies.

Two concepts of similarity are addressed and it is necessary to explain the terminology that is used in each case. Specific similarity is applied to the properties of the jet's turbulent concentration field that allow a collapse of its statistical measures

$\dagger$ Present address: Applied Physics Laboratory, University of Washington, Seattle, WA 98105, USA. 
with downstream distance at a fixed Reynolds number and Schmidt number. General similarity is used to describe properties of the jet's turbulent concentration field that are independent of Reynolds number.

The measured mean profile of jet fluid concentration is similar in general, and collapses on rays that emanate from the virtual origin of the jet (Wilson \& Danckwerts 1964; Becker, Hottel \& Williams 1967; Townsend 1976; Birch et al. 1978; Lockwood \& Moneib 1980; Chen \& Rodi 1980 and the references cited therein, plus figure 6). In the cylindrical coordinates used in this paper, this general similarity profile of the mean concentration of jet fluid, $\bar{C}$, takes the following form:

$$
\bar{C}(x, r)=\kappa \frac{C_{0} d^{*}}{x-x_{0}} g\left(\frac{r}{x-x_{0}}\right) \equiv \kappa \frac{C_{0}}{x} g(\eta),
$$

where $\kappa$ is a parameter determined by experiment, $d^{*}$ is the momentum diameter of the nozzle exit, $C_{0}$ is the jet exit concentration, $g(\eta)$ is a smooth function that has a maximum value of one at $\eta=0$ and is determined by experiment, $x$ is the distance from the jet nozzle along the axis of the jet, $r$ is the radial distance from the axis of the jet, and $x_{0}$ is the virtual origin of the jet flow in the far field. A schematic of this coordinate system is given as figure 1 .

The momentum diameter that appears in (1) is defined by

$$
d^{*}=\frac{2 \dot{m}_{0}}{\left(\pi \rho_{\infty} J_{0}\right)^{\frac{1}{2}}}
$$

where $\rho_{\infty}$ is the density of the reservoir fluid, $\dot{m}_{0}$ is the nozzle mass flux, and $J_{0}$ is the nozzle thrust. It was introduced in a limited way by Thring \& Newby (1953), used by Avery \& Faeth (1974), and modified to the present form by Dahm \& Dimotakis (1987). The momentum diameter has been used to collapse the results of a wide variety of jet experiments (see Dahm \& Dimotakis 1987). Note that $d^{*}$ reduces to the geometrical nozzle exit diameter, $d$, for density-matched jet and reservoir fluids and a perfect 'top-hat' exit velocity profile.

While the similarity of the mean profile is well supported by experimental results, there is substantial conflict between the results of different investigations regarding the similarity of concentration fluctuations in the turbulent jet (Chen \& Rodi 1980; Lockwood \& Moneib 1980; Dahm 1985). The reported discrepancies between investigations (see figure 10) could arise from many sources, including insufficient resolution of all of the fluctuating scales, contamination of the flow by buoyancy forces, unsteadiness in the jet source or quiescent reservoir, variations in molecular Schmidt number $\left(v_{\infty} / D_{\mathrm{j} \infty}\right)$ and Reynolds number between experiments, and differences in experimental configurations.

Recent work at a Reynolds number of 5000 by Dowling \& Dimotakis (1988) suggests that many statistical properties of the jet's fluctuating concentration field follow the same similarity law as the mean concentration. Their main finding was that the concentration field of the jet, downstream of $x / d=20$, was statistically selfsimilar in every detail along rays that emanate from the virtual origin of the jet. This paper examines the extent to which this concept of detailed similarity applies to turbulent jets at different Reynolds numbers. The available experimental information in liquid-phase jet flows is not as well developed as that of gas-phase flows. Therefore, the role of the Schmidt number in jet similarity is not addressed at length and is mentioned only where appropriate. The conclusions of this paper are based on comparisons of new and previously reported results for the mean concentration, $\bar{C}$, 


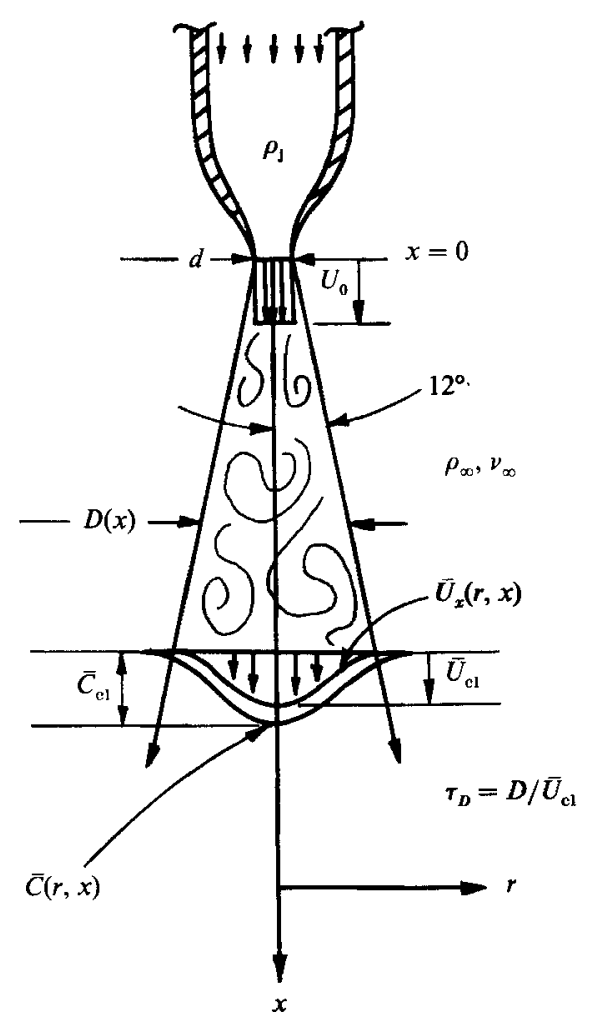

Figure 1. Turbulent jet coordinates.

the root-mean-square (r.m.s.) concentration fluctuation, $C_{\mathrm{rms}}^{\prime}$, the probability density function (PDF) of concentration, and the power spectrum of concentration fluctuations, $E_{c}(f)$. While this list of statistical parameters is certainly not exhaustive, it forms a useful basis for describing the fluctuating character of the jet flow.

The measurements reported here - single-point, concentration time-histories were made using a non-intrusive laser-Rayleigh scattering diagnostic in a steady, gasphase, axisymmetric, momentum-driven jet that issued into a large enclosure. The diagnostic was sensitive to the number-weighted concentration of jet gas molecules but the results are presented in terms of the mass-weighted concentration, since that is the quantity that best represents constant-density conditions (see Pitts 1986). Special effort was expended to eliminate and/or quantify the possible sources of experimental contamination mentioned above so that the natural state of the jet's turbulent concentration field could be investigated. These precautions are summarized at the beginning of the next section.

The new results are presented at three jet exit Reynolds numbers : 5000,16000 and 40000. These Reynolds numbers were computed from the jet exit velocity, $U_{0}$ (taken as uniform across the nozzle exit), the geometrical nozzle exit diameter, $d$, and the reservoir kinematic viscosity, $\nu_{\infty}$, i.e.

$$
R e_{0}=\frac{U_{0} d}{\nu_{\infty}}
$$

The experimental design, apparatus, and diagnostic technique are described in the 
next section. They are the same ones employed by Dowling \& Dimotakis (1988), and are described in detail in Dowling (1988), and Dowling, Lang \& Dimotakis (1989). The experimental findings, with comparisons and discussion, are presented in $\S 3$. The conclusions are given in $\S 4$.

It is worth emphasizing that the results and comparisons presented in $\$ 3$ are specialized to the concentration field of the round, momentum-driven, free turbulent jet. Comparisons of the current results with concentration or velocity measurements from other shear flows, which do not possess the same special similarity properties as the jet, are not included. Additionally, the chosen allocation of resources did not allow for measurements of both concentration and velocity in a manner that was suitable, so the current experiments can only be considered to address properties of the jet's velocity field which can be inferred from concentration measurements. The only velocity scale, besides $U_{0}$, that is referenced throughout the manuscript is the mean centreline velocity, $\bar{U}_{\mathrm{cl}}$, which was computed from the empirical decay law suggested by Chen \& Rodi (1980). As discussed in $\$ 3.2$, this does not affect the conclusions drawn from this research, nor impose any significant penalty on the presentation of the results.

\section{Experimental design, apparatus, and technique}

The experiment was designed to resolve the entire range of spatial and temporal concentration scales while mitigating the influences from the previously mentioned contamination sources. The spatial resolution requirement was that the measurement volume had to be smaller than the local Batchelor scale, $\lambda_{\mathrm{B}}$ (Batchelor 1959), in each dimension. The temporal resolution requirement was that the bandwidth of the data acquisition system had to comfortably exceed the mean passage frequency, $f_{\mathrm{B}}$, of lengthscales the size of $\lambda_{B}$. Actual lengths and frequencies were estimated, for design purposes, from classical turbulence formulae and published experimental results.

For the chosen gas pairs, the Schmidt number, $S c$, was near unity so the Batchelor and Kolmogorov scales were assumed to be about equal (Batchelor 1959; Monin \& Yaglom 1975).

$$
\lambda_{\mathrm{B}} \equiv \frac{\lambda_{\mathrm{K}}}{S c^{\frac{1}{2}}} \approx \lambda_{\mathrm{K}}=\beta\left\{\frac{\nu_{\infty}^{3}}{\bar{\epsilon}}\right\}^{\frac{1}{4}} \propto D \operatorname{Re}_{0}^{-\frac{3}{4}} .
$$

Here, $\lambda_{\mathrm{K}}$ is the Kolmogorov scale, $\beta$ is a dimensionless constant, $\bar{\epsilon}$ is the mean energy dissipation rate, and $D$ is the local jet diameter (see figure 1). Actual values of $\lambda_{\mathbf{B}}$ used to design the experiment were calculated using the results of Friehe, Van Atta \& Gibson (1971) for the mean centreline energy dissipation rate in the jet,

$$
\bar{\epsilon}=48 \frac{U_{0}^{3}}{d}\left(\frac{x-x_{0}}{d}\right)^{-4}
$$

and a conservative value of $\beta(=1)$. The mean Batchelor-scale passage frequency, $f_{\mathrm{B}}$, was calculated from the mean centreline velocity decay law suggested by Chen \& Rodi (1980) and (4), i.e.

where

$$
f_{\mathrm{B}} \approx \frac{\bar{U}_{\mathrm{cl}}}{\lambda_{\mathrm{K}}}=\bar{U}_{\mathrm{cl}}\left(\frac{\bar{\epsilon}}{\nu_{\infty}^{3}}\right)^{\frac{1}{4}},
$$

The effect of buoyancy forces was estimated using the buoyancy-lengthscale 
formalism developed in Fischer et al. (1979). The measurements of Papanicolaou \& List $(1987,1988)$ show that the jet is momentum dominated for $x / l_{\mathrm{b}}$ less than one; $l_{\mathrm{b}}$ is a buoyancy lengthscale defined by

where

$$
\begin{gathered}
l_{\mathrm{b}}=\left(\frac{\left(J_{0} / \rho_{\infty}\right)^{3}}{\left(B / \rho_{\infty}\right)^{2}}\right)^{\frac{1}{4}}, \\
B=\int_{A_{0}}\left(\rho_{\mathrm{j}}-\rho_{\infty}\right) U_{\mathrm{j}} \mathrm{d} A,
\end{gathered}
$$

$\rho_{\mathrm{j}}$ is the density of pure jet gas, $U_{\mathrm{j}}$ is the jet nozzle exit velocity profile, and $A_{0}$ refers to the jet nozzle exit area (for a 'top-hat' exit velocity profile $U_{j}=U_{0}$ ). Chen \& Rodi (1980) propose a more restrictive criterion of $x / l_{\mathrm{b}} \leqslant 0.53$, for momentum-dominated flow. The present measurements all satisfy $x / l_{\mathrm{b}} \leqslant 0.4$.

To accommodate the diagnostic chosen, the experiments were performed inside a special enclosure. To prevent reverse flow inside this chamber, a mild coflowing velocity was imposed on the reservoir gas. The effect of this mild coflow on the evolution of the jet was estimated from the results of Reichardt (1964) who measured the velocity half-width of coflowing jets as a function of $x / l_{\mathrm{c}}$, where $l_{\mathrm{c}}$ is the momentum lengthscale associated with a jet in a coflowing stream,

$$
l_{\mathrm{c}}^{2}=\frac{4}{\pi \rho_{\infty} U_{\mathrm{c}}^{2}}\left\{\int_{A_{0}} \rho_{\mathrm{j}}\left(U_{\mathrm{j}}-U_{\mathrm{c}}\right) U_{\mathrm{j}} \mathrm{d} A\right\}
$$

(also see Hinze 1975). Reichardt's work suggests that the coflow has little effect on the jet for $x / l_{\mathrm{c}}$ less than about one. All of the present measurements satisfy $x / l_{\mathrm{c}} \leqslant \mathbf{0 . 5}$.

To assure that the jet and coflow were stable during an experiment; the measured mean concentration was checked during the first, middle, and final thirds of a run to make sure that there was less than $\pm 10 \%$ to $20 \%$ variation. The repeatability of runs was also checked and found satisfactory. To ensure that adequate statistics were collected, an experiment was required to last many times longer than the mean convection time of the local jet diameter. This timescale, $\tau_{D}$, was estimated from the half-angle of the jet's turbulent cone, about $12^{\circ}$ (White 1974), and (7):

$$
\tau_{D}=D(x) \bar{U}_{\mathrm{cl}}^{-1}, \quad \text { where } D(x)=2\left(x-x_{0}\right) \tan \left(12^{\circ}\right) \approx 0.43\left(x-x_{0}\right) .
$$

The time interval of data collection varied from $16 \tau_{D}$ to $180 \tau_{D}$. This sampling interval proved to be adequate for the central region of the jet, but may not have been long enough to accurately determine the statistics of the flow near the edges of the jet where the mean velocity is smaller, the relevant convection timescale is longer than $\tau_{D}$, and the flow is intermittent.

The Schmidt number for each of the gas pairs used was estimated from the empirical correlations for the diffusivity of gases found in Reid, Prausnitz \& Sherwood (1977). These correlations are accurate to within about 5 or $10 \%$.

The three Reynolds numbers explored here were chosen in accordance with the performance of the experimental apparatus and diagnostic. At $R e_{0}=5000$, all of the design criteria were met and measurements were made at $x / d=20,40,60$, and 80 . However, these results showed that the resolution criteria were conservative and had been met by a very wide margin. The second Reynolds number, 16000, was chosen to take better advantage of the diagnostic capabilities while still resolving all of the fluctuating concentration scales in the flow at different downstream distances from the jet nozzle. At $R e_{0}=16000$, measurements were made at $x / d=30$ and 90 . The 


\begin{tabular}{|c|c|c|c|c|}
\hline \multicolumn{5}{|c|}{ (a) For $R e_{0}=5000\left(x_{0}=-3.7 d\right)$} \\
\hline & $x / d=20$ & $x / d=\mathbf{4 0}$ & $x / d=60$ & $x / d=80$ \\
\hline$\lambda_{\mathrm{B}}(\mu \mathrm{m})$ & $(290)$ & $(530)$ & $(780)$ & $(1020)$ \\
\hline Diode size $(\mu \mathrm{m})$ & 200,500 & 500 & 500 & 1000 \\
\hline$f_{\mathrm{B}}(\mathrm{kHz})$ & (3.7) & $(1.08)$ & $(0.51)$ & $(0.29)$ \\
\hline Digitization rate $(\mathrm{kHz})$ & 96,40 & 20 & 10 & 5 \\
\hline$\tau_{D}(\mathrm{~s})$ & $(0.18)$ & $(0.62)$ & $(1.31)$ & $(2.3)$ \\
\hline \multirow[t]{3}{*}{ No. of $\tau_{D}$ during a run } & 40,100 & 40 & 30 & 16 \\
\hline & \multicolumn{2}{|c|}{$\begin{array}{l}\text { (b) For } R e_{0}=16000 \\
\left(x_{0}=0.5 d\right)\end{array}$} & \multirow{2}{*}{\multicolumn{2}{|c|}{$\begin{array}{l}\text { (c) For } R e_{0}=40000 \\
\left(x_{0} \text { taken to be zero) }\right. \\
x / d=60\end{array}$}} \\
\hline & $x / d=30$ & $x / d=90$ & & \\
\hline$\lambda_{\mathrm{B}}(\mu \mathrm{m})$ & $(60)$ & $(180)$ & \multicolumn{2}{|c|}{$(61)$} \\
\hline Diode size $(\mu \mathrm{m})$ & 200 & 500 & \multicolumn{2}{|c|}{200} \\
\hline$f_{\mathrm{B}}(\mathrm{kHz})$ & (99) & $(10.9)$ & \multicolumn{2}{|c|}{ (120) } \\
\hline Digitization rate $(\mathrm{kHz})$ & 180 & 30 & \multicolumn{2}{|c|}{240} \\
\hline$\tau_{p}(\mathrm{~s})$ & $(0.016)$ & $(0.146)$ & \multicolumn{2}{|c|}{$(0.026)$} \\
\hline No. of $\tau_{D}$ during a run & 180 & 120 & \multicolumn{2}{|c|}{80} \\
\hline
\end{tabular}

TABLE 1. Resolution and parameter summary at each Reynolds number. Calculated values appear in parentheses.

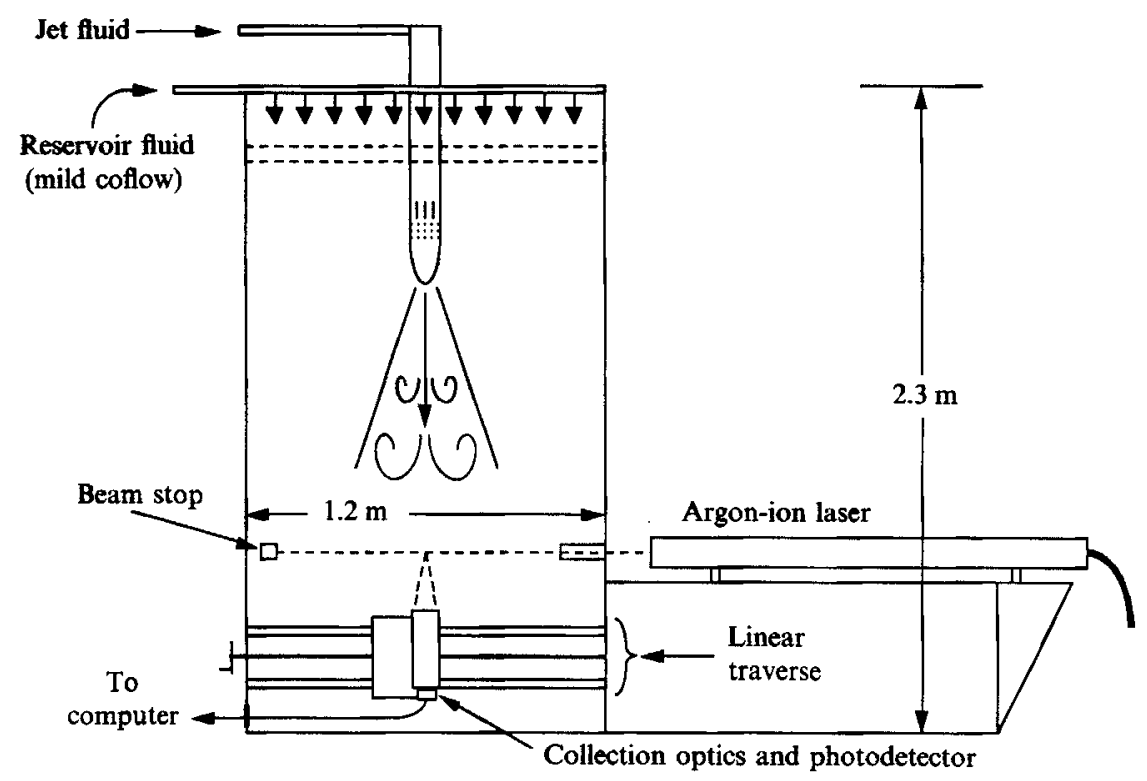

Figure 2. Schematic of the experimental apparatus in cross-section.

final Reynolds number, 40000 , was the highest at which data could be collected with the present experimental arrangement while still meeting the resolution requirements. This meant that only one downstream location was explored, $x / d=60$. A summary of the calculated and actual resolution of each set of experiments is given in table 1. The Taylor Reynolds number, $R e_{\lambda}$, along the centreline of the jet was estimated from the formulae in Hinze (1975), (5) and (7), and a typical value of the 
centreline r.m.s. velocity fluctuation, $u_{\text {rms }}^{\prime} \approx 0.25 \bar{U}_{\mathrm{cl}}$. The results are: $R e_{\lambda} \approx 100$, 180,280 for $R e_{0}=5000,16000$, and 40000 respectively.

The experiments were conducted in a large enclosure $(1.2 \times 1.2 \times 2.3 \mathrm{~m})$ that was used to isolate the jet and reservoir gases from dust particles, laboratory lighting, and air currents (see figure 2 ). The jet was produced by a vertically adjustable nozzle with $d=19.0 \mathrm{~mm}$ for the experiments at $R e_{0}=5000$, and $d=7.62 \mathrm{~mm}$ for the experiments at $R e_{0}=16000$ and 40000 . Both nozzles had smooth inner contours and their measured exit turbulence levels were less than $0.2 \%$ at the experiment Reynolds numbers. A uniform coflowing velocity, typically less than 0.006 of the jet exit velocity was produced over the entire cross-section of the large enclosure. The volume flux of the coflow was chosen to provide the jet entrainment needs to a point below the farthest measuring station (Ricou \& Spalding 1961). Plexiglas windows $(1 \times 1.5 \mathrm{~m})$ were used for shadowgraph imaging of the jet. The exhaust gases exited through the bottom of the enclosure.

For the experiments at $R e_{0}=5000$, the jet gas was ethylene, $\mathrm{C}_{2} \mathrm{H}_{4}$, and the reservoir gas was nitrogen. This gas pair has a jet/reservoir density ratio of 1.0015 and a molecular Schmidt number of 1.0. For the experiments at $R e_{0}=16000$ and 40000 , the jet gas was propylene, $\mathrm{C}_{3} \mathrm{H}_{6}$, and the reservoir gas was argon. This gas pair has a jet/reservoir density ratio of 1.053 and a Schmidt number of 1.2 . In both cases, the dynamic head at the nozzle exit was measured to determine $U_{0}$. An axisymmetric boundary-layer calculation was used to estimate boundary-layer thicknesses at the nozzle exit in order to calculate $d^{*}$ for each Reynolds number. The results were $d^{*}=0.96 d$ at $R e_{0}=5000, d^{*}=1.005 d$ at $R e_{0}=16000$, and $d^{*}=1.01 d$ at $R e_{0}=40000$.

Laser-Rayleigh scattering was used to determine the instantaneous concentration of the binary mixture of jet and reservoir gases in a small focal volume within the turbulent cone of the jet. Laser-Rayleigh scattering has been successfully used and documented in many previous studies (see discussion and citations in Dowling et al. 1989), and is only described briefly here. The technique makes use of the fact that gas molecules elastically scatter photons, and that different molecules have different Rayleigh-scattering cross-sections. Rayleigh scattering from a binary gas mixture can be described by an extinction coefficient, $\alpha_{\mathrm{T}}$, that is related to the mole fraction, $X_{1}$, of one of the gases of the mixture,

$$
\alpha_{T}=\alpha_{1} X_{1}+\alpha_{2}\left(1-X_{1}\right)
$$

where $\alpha_{1}$ and $\alpha_{2}$ are the extinction coefficients of the pure gases which compose the binary mixture under consideration. The amount of scattered light that can be collected, and converted to a single current using a photodetector, is proportional to $\alpha_{\mathrm{T}}$. Hence, the magnitude of the detected current is related in a linear manner to $X_{1}$. In practice, the two end points, $X_{1}=0$ and 1 , of this linear relationship are recorded for the purposes of calibration, and the conversion from signal current to mole fraction is accomplished by linear interpolation.

For these experiments, the Rayleigh-scattered light from a short segment of a nominally $20 \mathrm{~W}$ argon-ion laser beam was imaged one-to-one onto a small aperture photodiode. The diameter of the sensitive area of the photodiode was between 0.20 and $1.00 \mathrm{~mm}$; the local resolution requirements of the jet dictating the size used for each experimental run (see table 1). The signal current from the photodiode was amplified, filtered, and sent to an LSI-PDP-11/73 based computer system where it was digitized and stored for subsequent processing. The sampling frequency and filter bandwidth were chosen to ensure that the temporal resolution requirements 


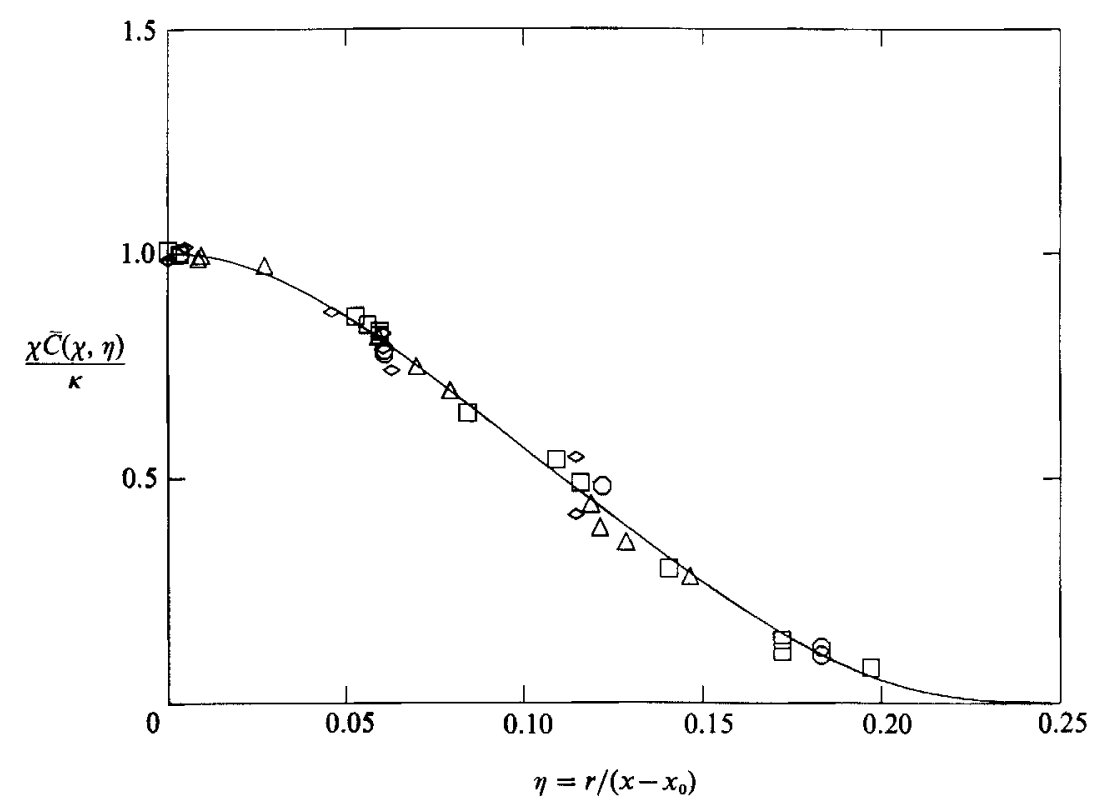

Figure 3. Scaled mean concentration (using the parameters of $(1)), \chi \bar{C}(\chi, \eta) / \kappa$, versus the scaled radial coordinate, $\eta=r /\left(x-x_{0}\right)$, at $R e_{0}=5000$ with $\kappa=5.11, x_{0}=-3.7 d$, and $d^{*}=0.96 d$ $(\square, x / d=20 ; 0,40 ; \triangle, 60 ; \diamond, 80)$.

were easily met in all cases, with the possible exception of the highest Reynolds number runs.

The experimental methodology was quite simple in principle. The large enclosure was filled with pure reservoir gas. When residual velocities from the filling process had dissipated, the coflow and the jet were started together. The start of data acquisition was delayed until the jet had established a steady state. Runs lasted from a few seconds to almost a minute. The total number of individual concentration measurements in a single run was typically $2^{19}(>500000)$. The sensitivity of the whole system was calibrated before and/or after each run by introducing pure jet and reservoir gases into the focal volume. The absolute accuracy of the calibration was about \pm 1 parts per $10^{3}$, which resulted from mild drift in the laser power and electronics. The techniques used for data processing and data reduction are described in Dowling (1988), and Dowling et al. (1989).

\section{Results}

\subsection{Basic properties of the concentration field of the jet}

The mean value of concentration from each run was computed by simple averaging, and plotted using the axis scaling suggested by (1). The results are displayed on figure 3 for the data at $R e_{0}=5000$ with $\kappa=5.11 \pm 0.05$ and $x_{0}=-3.7 d$, on figure 4 for the data at $R e_{0}=16000$ with $\kappa=4.73 \pm 0.1$ and $x_{0}=0.5 d$, and on figure 5 for the data at $R e_{0}=40000$. It was not possible to obtain precise values for $\kappa$ and $x_{0}$ at $R e_{0}=40000$ because measurements were only made at $x / d=60$, so there is no scaling of the vertical axis on figure 5. The value of $\kappa$ at $R e_{0}=40000$ is estimated to be between 5.1 and 5.2. It is important to note that the axis scaling on figures 3 and 4 is based only on the fitted values of $\kappa$ and $x_{0}$ at each Reynolds number. There is no 


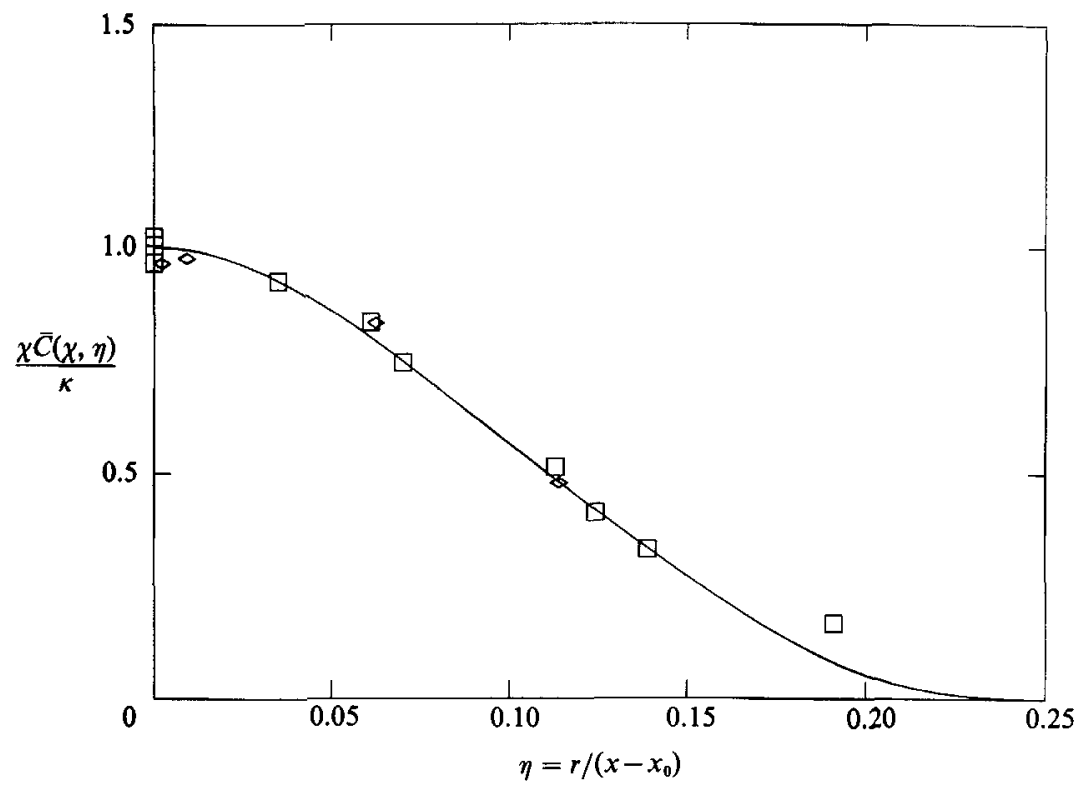

Figure 4. Scaled mean concentration (using the parameters of $(1)$ ), $\chi \bar{C}(\chi, \eta) / \kappa$, versus the scaled radial coordinate, $\eta=r /\left(x-x_{0}\right)$, at $R e_{0}=16000$ with $\kappa=4.73, x_{0}=0.5 d, d^{*}=1.005 d$, and the fitted mean profile from the data at $R e_{0}=5000(\square, x / d=30 ; \diamond, 90)$.

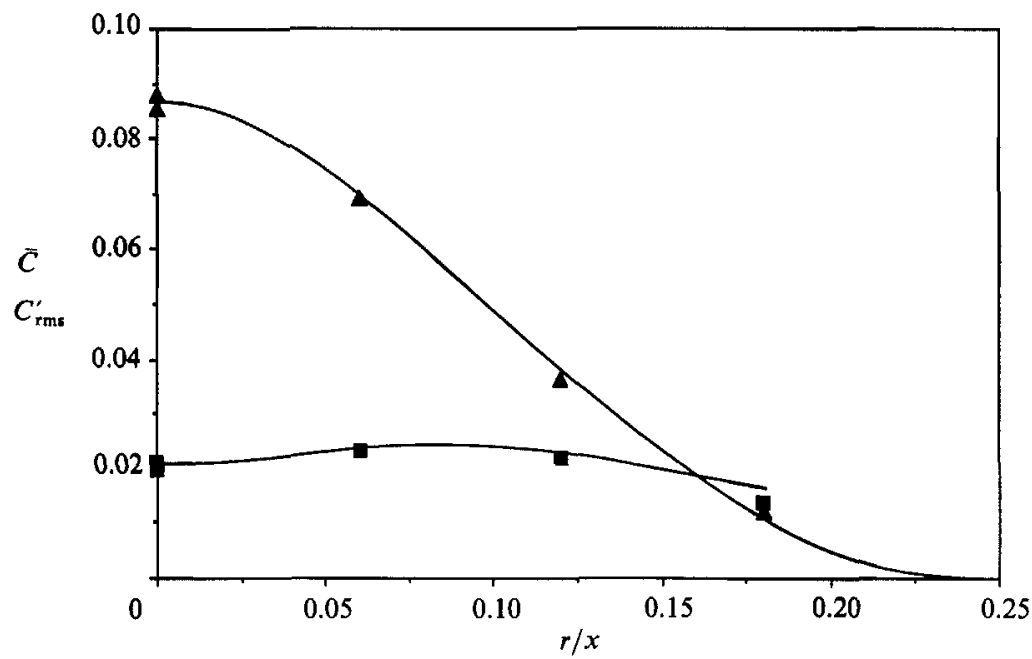

Figure 5. Mean (A) and r.m.s. ( $)$ concentration at $R e_{0}=40000$ and $x / d=60$, with fitted mean profile from the data at $R e_{0}=5000$, and r.m.s. profile from the data at $R e_{0}=16000$.

'self-normalization' of the data on either of these figures. For example, separate normalizations by the local centreline mean, or concentration profile radius at halfmaximum were not necessary. The fact that the profile function $g(\eta)$ emerges in these plots testifies to the specific similarity of $\bar{C}$, and the proper selection of $\kappa$ and $x_{0}$ at each Reynolds number. Figures 3,4 and 5 show that the experimental apparatus and technique produced a turbulent jet with the accepted general similarity form for $\bar{C}$. The mean concentration profile curve on figures 3,4 , and 5 is a least-squares fit to the data at $R e_{0}=5000$. 


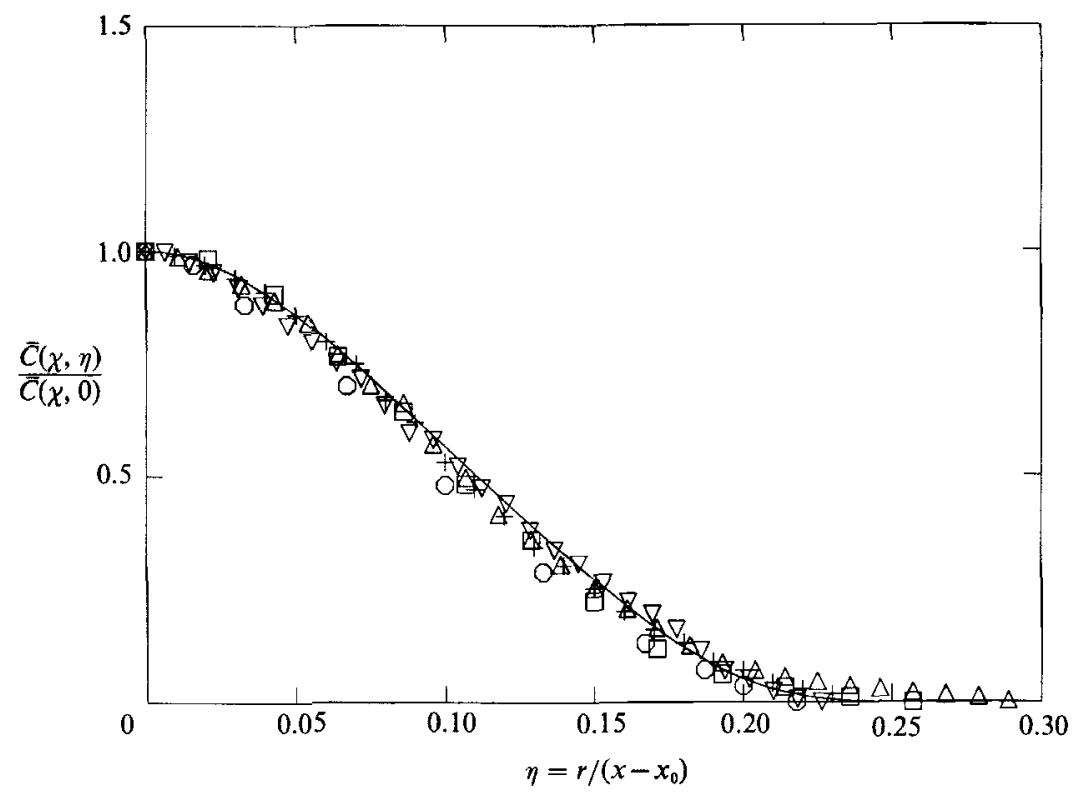

Fiaure 6. Comparison of the fitted mean concentration profile, $\bar{C}(\chi, \eta) / \bar{C}(\chi, 0)$, with the results of other experiments $(O$, Lockwood \& Moneib 1980; $\square$, Corrsin \& Uberoi 1950; $\triangle$, Birch et al. 1978; + , Becker et al. 1967; $\nabla$, Dahm 1985; - , current profile).

\begin{tabular}{|c|c|c|c|}
\hline Author & $R e_{0}$ & $S c$ or $\operatorname{Pr}$ & Diagnostic \\
\hline Becker et al. (1967) & 54000 & 38000 & Smoke scattering \\
\hline Birch et al. (1978) & 16000 & 0.70 & Raman scattering \\
\hline Clay $(1973)$ & 800000 & 0.70 & Thermometry (air) \\
\hline Corrsin \& Uberoi (1950) & 33000 and up & 0.7 & Thermometry (air) \\
\hline Dahm (1985) & 5000 & $600-800$ & Laser-induced fluorescence \\
\hline Lockwood \& Moneib (1980) & 50000 & 0.7 & Thermometry (air) \\
\hline Wilson \& Danckwerts (1964) & $20000-60000$ & 0.7 & Thermometry (air) \\
\hline Papanicolaou \& List (1987) & $10000-16000$ & 7.0 & Thermometry $\left(\mathrm{H}_{2} \mathrm{O}\right)$ \\
\hline Papanicolaou \& List (1988) & $2600-3600$ & $\approx 10^{3}$ & Laser-induced fluorescence \\
\hline Papantoniou (1985) & 5600 & $600-800$ & Laser-induced fluorescence \\
\hline \multirow{3}{*}{ Present study } & 5000 & 1.0 & Rayleigh scattering \\
\hline & 16000 & 1.2 & \\
\hline & 40000 & 1.2 & \\
\hline
\end{tabular}

Table 2. Turbulent jet mixing experiments

A comparison of this fitted profile with the published profiles of other experiments is given on figure 6 . The agreement between profiles is good and the small differences can probably be attributed to the differing experimental conditions and techniques of each experiment. Table 2 lists some of the important parameters of the experiments used for comparison with the current studies. All of these studies are of turbulent jets beyond $x / d=20$, above $R e_{0}=2500$, and estimated to be free of serious influence from buoyancy forces or a coflowing stream. Detailed comparison with any larger body of work is beyond the scope of this paper.

A comparison of the current and previous experimental results for the mean centreline concentration, $\bar{C}(\chi, 0)$, is provided on figure 7 . The vertical axis is scaled so that the measured data will fall on horizontal lines when the far-field behaviour 


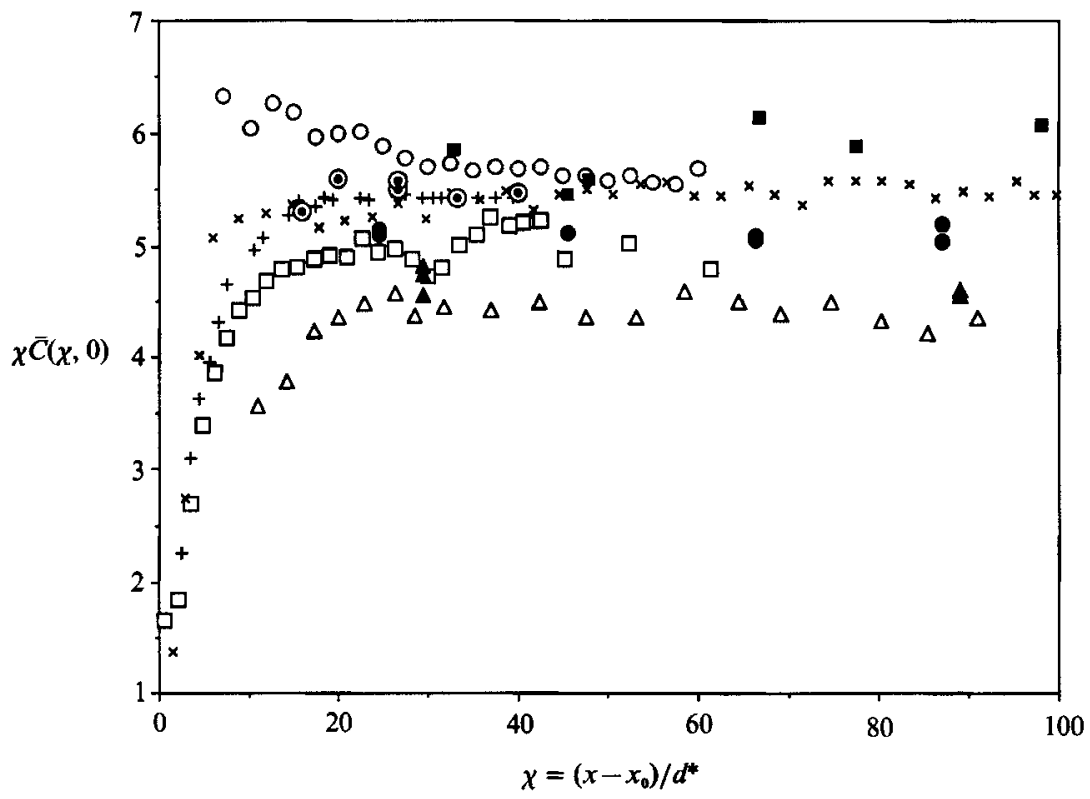

FigURE 7. Comparison of current results with the results of previous experiments for the scaled mean centreline concentration, $\chi \bar{C}(\chi, 0)$, versus the scaled downstream coordinate, $\chi=\left(x-x_{0}\right) / d^{*}$ ( $\square$, Lockwood \& Moneib 1980; O, Wilson \& Dunckwerts 1964; $\triangle$, Birch et al. 1978; +, Becker et al. 1967, $\times$, Dahm 1985; $\odot$, Papanicolaou \& List 1987; $\square$, Papanicolaou \& List 1988; - present data $R e_{0}=5000 ; \Delta$, present data $R e_{0}=16000$ ).

of the jet is attained. The ordinate of the horizontal portion of the curves is the value of $\kappa$ for each experimental effort. The current data fall within the scatter of the results of the other experiments.

The reported variation in $\kappa$ is, perhaps, interesting and unexpected. It could result from improper calculation of $d^{*}$, miscalibration of experimental diagnostics, or Reynolds-number effects. For example, the value of $\kappa$ differs by about $8 \%$ in the current studies at 5000 and 16000 . Different nozzles were used for each set of experiments. It is possible that the design of the smaller nozzle could have caused the maximum momentum flux to occur slightly beyond the nozzle's exit plane in an effective area smaller than $\frac{1}{4} \pi d^{2}$ (vena contracta). This would produce a slightly smaller value of $d^{*}$ than the result of the axisymmetric boundary-layer calculation. A correction of this type would bring the current results for $\kappa$ closer together. It is unlikely, however, that miscalculation and/or miscalibration could account for the entire range of variation in $\kappa$ seen on figure $7(4<\kappa<6)$. Reynolds-number effects cannot be ruled out, but an examination of table 2 and figure 7 does not reveal a simple monotonic trend in $\kappa$ with $R e_{0}$. Other possible sources for the reported variation in $\kappa$ can be ruled out. Misalignment of measurement points with respect to the true jet axis could cause the reported discrepancies, but to account for the $8 \%$ change in $\kappa$ the misalignment would have to be about $\pm 1.5^{\circ}$, almost an order of magnitude greater than the estimated angular location error (about $\pm 0.2^{\circ}$ ) of the current experiments. Initial density differences between the jet and reservoir fluids are accounted for in the far field through $d^{*}$. Additionally, there does not appear to be a physical mechanism which would allow differences in the Schmidt (Prandtl) number between experiments to change the jet's bulk entrainment rate of reservoir fluid, and thus influence the value of $\kappa$. At low and moderate Reynolds numbers, 


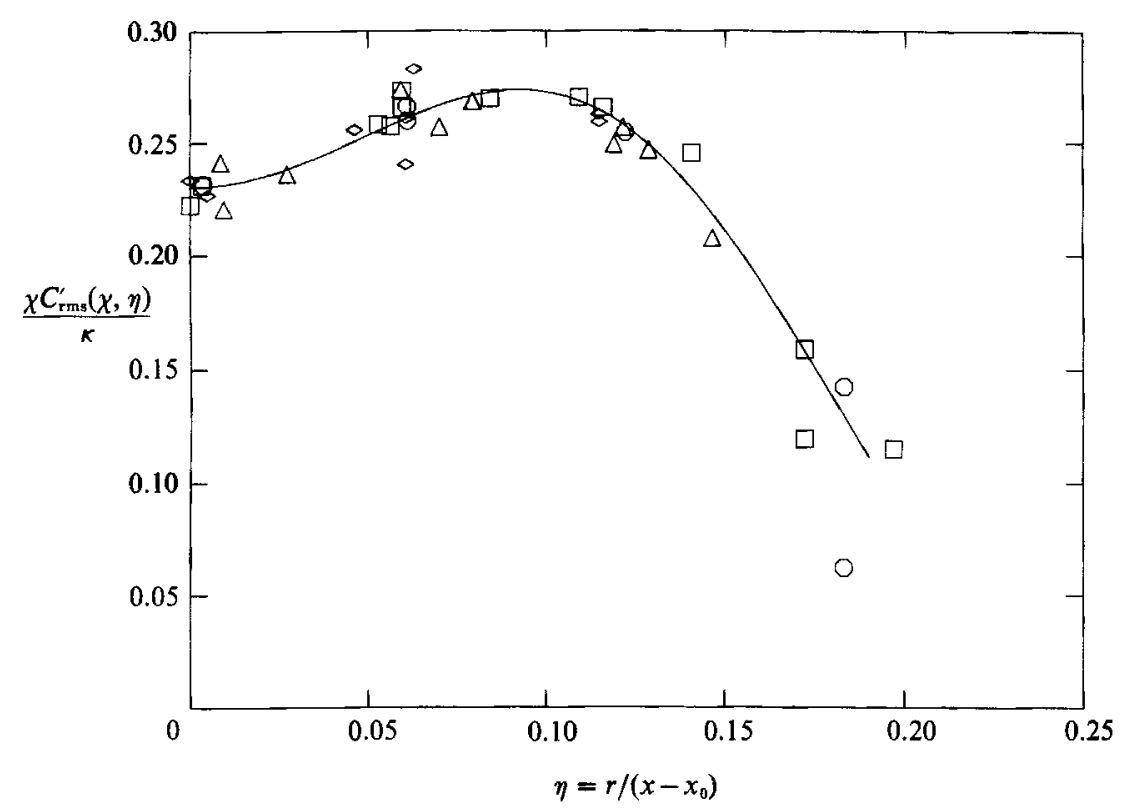

Figure 8. Root-mean-square concentration fluctuation level plotted versus $\eta=r /\left(x-x_{0}\right)$, with the same scaling of the vertical axis that collapses the mean concentration at $R e_{0}=5000(\square, x / d=20$; $0,40 ; \triangle, 60 ; \diamond, 80)$.

Niwa et al. (1984) have demonstrated the dependence of $\kappa$ on $R e_{0}$. The recent article on self-preservation in turbulent flows by George (1989) suggests that details of the jet nozzle exit conditions play a role in determining $\kappa$.

The root-mean-square (r.m.s.) concentration fluctuation level, $C_{\mathrm{rms}}^{\prime}$, was computed directly from each time series after optimal filtering (Wiener 1949, described in Press et al. 1986, illustrated in Dowling et al. 1989). This filtering was performed to remove noise from the measured time series. For any of the current data sets, the difference in the computed value of $C_{\text {rms }}^{\prime}$ before and after optimal filtering was a small percentage $\left(\approx 0.1 \%\right.$ and always $<2 \%$ ) of the final value of $C_{\mathrm{rms}}^{\prime}$ computed after filtering. The results for $C_{\mathrm{rms}}^{\prime}$ are plotted on figures 5, 8 and 9 for $R e_{0}=40000,5000$ and 16000 , respectively. The axis scaling employed on figures 8 and 9 is exactly the same as that used on figures 3 and 4 for $\bar{C}$ at the same Reynolds numbers. As before, no 'self-normalization' of the data was necessary. Note that the vertical axes on figures 8 and 9 are not scaled by the centreline r.m.s. fluctuation level. The profile curve for $C_{\mathrm{rms}}^{\prime}$ on figure 5 is the same as the one on figure 9 . The fact that the r.m.s. fluctuation level data cluster about a single curve, in the specific similarity coordinates of the mean concentration, at $R e_{0}=5000$, and 16000 , implies that $C_{\text {rms }}^{\prime}$ and $\bar{C}$ conform to the same specific similarity law, and that $C_{\mathrm{rms}}^{\prime} / \bar{C}$ is a general similarity variable for the jet. This issue is further addressed in the next section.

As noted in the Introduction, this behaviour was not found in many previous investigations. Figure 10 is a plot of $C_{\mathrm{rms}}^{\prime} / \bar{C}$ on the centreline of the jet for several experiments. The present data at $R e_{0}=5000$ and 16000 fall on horizontal lines, indicating that $C_{\mathrm{rms}}^{\prime}$ and $\bar{C}$ follow the same specific similarity laws based on the values of $\kappa$ and $x_{0}$ at each Reynolds number. The value of $C_{\mathrm{rms}}^{\prime} / \bar{C}$ on the centreline for the current studies is $0.230 \pm 0.007$ at $R e_{0}=5000,0.237 \pm 0.005$ at $R e_{0}=16000$, and $0.23 \pm 0.01$ at $R e_{0}=40000$. The failure of many previous studies to find a constant value of $C_{\mathrm{rms}}^{\prime} / \bar{C}$ on the centreline of the jet may be attributable to 


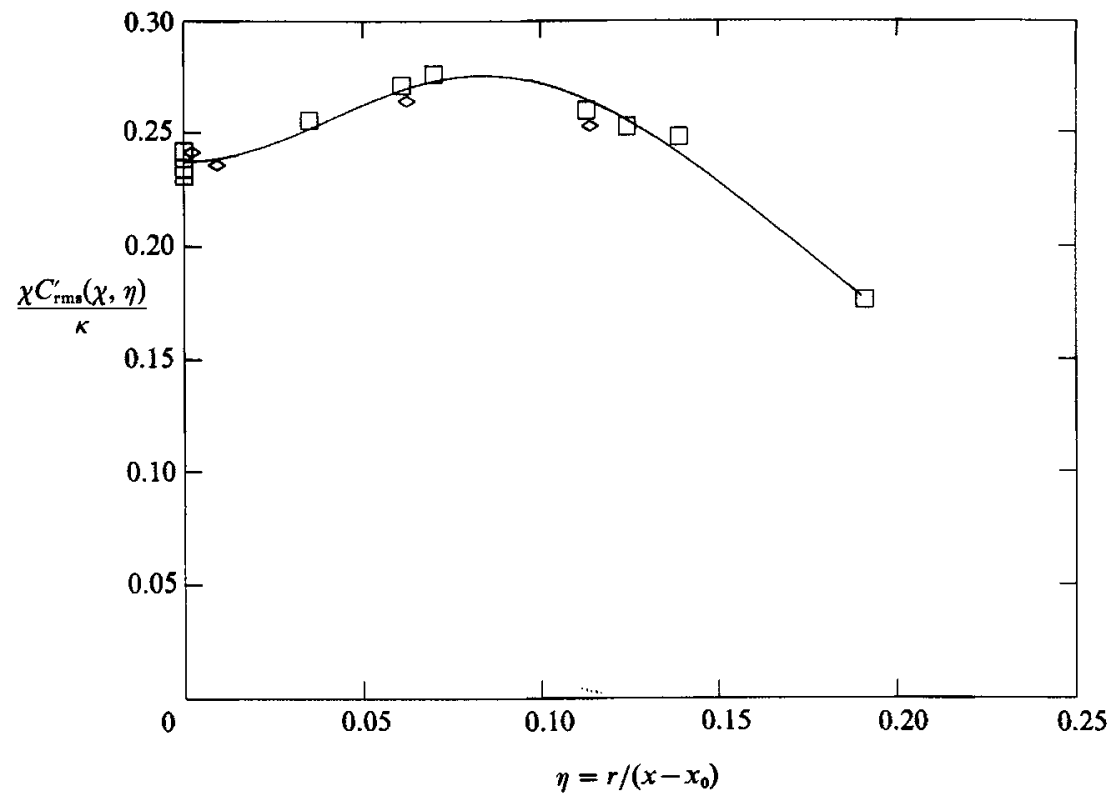

FiguRe 9. Root-mean-square concentration fluctuation level plotted versus $\eta=r /\left(x-x_{0}\right)$, with the same scaling of the vertical axis that collapses the mean concentration at $R e_{0}=16000$ ( $\square$, $x / d=30 ; \diamond, 90)$.

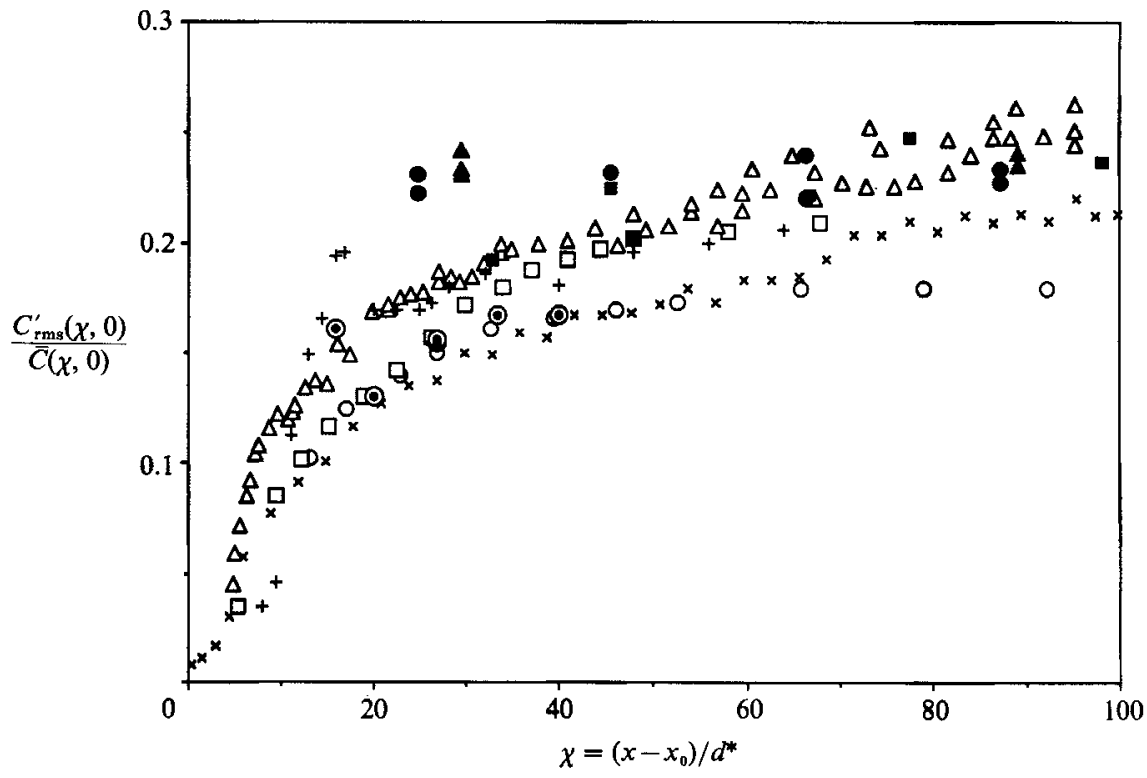

FiguRe 10. Comparison of current results with the results of previous experiments for the centreline ratio of the r.m.s. to mean concentration, $C_{\mathrm{rms}}^{\prime}(\chi, 0) / \bar{C}(\chi, 0)$, versus the scaled downstream coordinate, $\chi=\left(x-x_{0}\right) / d^{*}(\square$, Lockwood \& Moneib 1980; $O$, Wilson \& Danckwerts $1964 ; \triangle$, Birch et al. 1978; +, Becker et al. 1967; $\times$, Dahm 1985; $\odot$, Papanicolaou \& List 1987; . Papanicolaou \& List 1988; - , present data $R e_{0}=5000 ; \Delta$, present data $R e_{0}=16000$ ). 


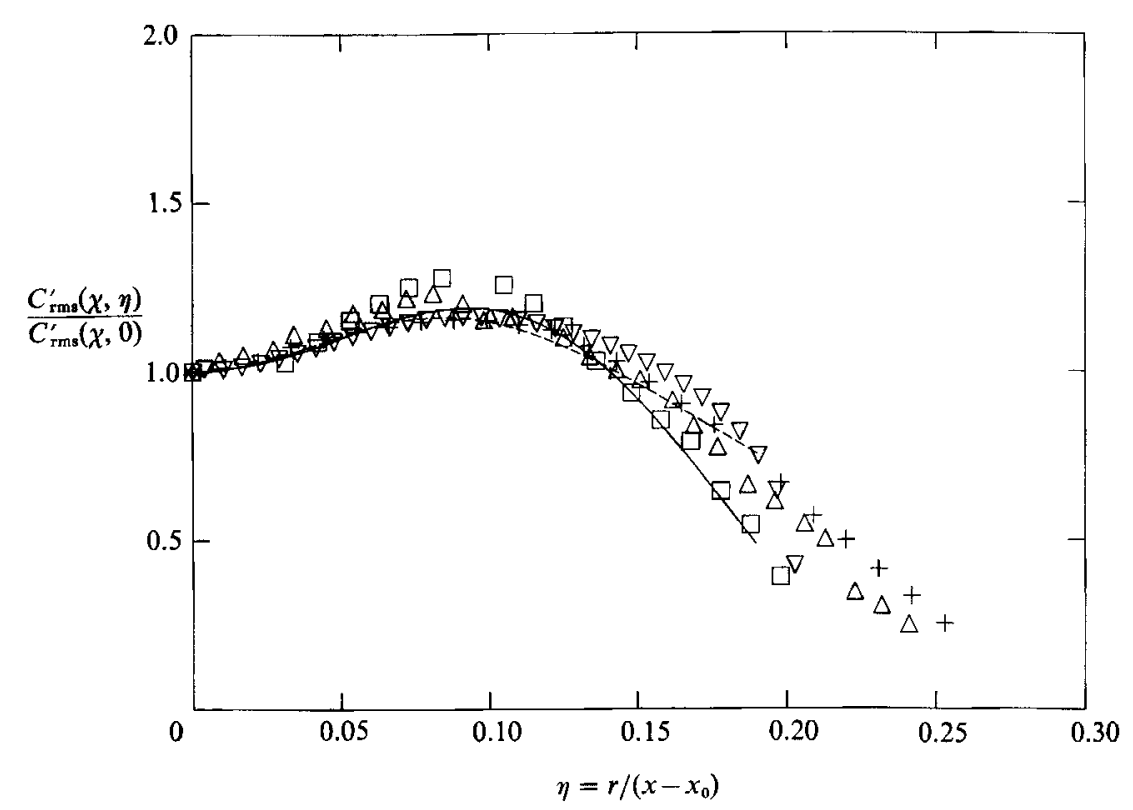

FIGURE 11. Comparison of the current results with the results of previous experiments for the r.m.s. concentration fluctuation level, divided by its centreline value, versus $r /\left(x-x_{0}\right)(\square$, Lockwood \& Moneib $1980 ; \triangle$, Birch et al $1978 ;+$, Becker et al $1967 ; \nabla$, Dahm $1985 ;-$, current profile at $R e_{0}=5000 ;---$, current profile at $R e_{0}=16000$ ). Reynolds and Schmidt number information for the plotted data is available in table 2 .

inadequate temporal, spatial, or dynamic resolution of concentration fluctuations nearer to the jet nozzle. Because the process of computing an r.m.s. does not commute with averaging, a measurement probe that performs a fixed amount of averaging in time or space may not produce consistent r.m.s. measurements when placed in a flow with resolution requirements that vary with downstream distance. For the jet, $f_{\mathrm{B}}$ is proportional to $\left(x-x_{0}\right)^{-2}$, and $\lambda_{\mathrm{B}}$ is proportional to $\left(x-x_{0}\right)$; as a result, the resolution requirements are more difficult to satisfy at lower values of $\left(x-x_{0}\right) / d$. Additionally, if the measurement noise is large enough to obscure a significant portion of the signal's power spectrum, then filtering this noise may lead to an underestimation of $C_{\mathrm{rms}}^{\prime}$ because part of the signal spectrum may be filtered too. Recall that $C_{\mathrm{rms}}^{\prime}$ is the square root of the area under the power spectral curve (see (15) for the definition used in this study). If the noise is not filtered, then $C_{\mathrm{rms}}^{\prime}$ may be overestimated because it may include contributions from the noise. The spatial and temporal resolution of the present experiments was adjusted at each downstream measurement location to prevent direct resolution difficulties, and the measurement diagnostic had sufficient dynamic range to effectively eliminate the influence of noise. Concentration fluctuation power spectra of the current data are described in \$3.2, and the results presented there support these contentions concerning the resolution of the current measurements.

If the fluctuation levels of all the experiments are normalized by their centreline value, the r.m.s. profiles can be compared. Figure 11 is a plot of the fitted curves from this study with the results from other experiments. The less than perfect collapse is not surprising since the six experiments shown were performed at different Reynolds and Schmidt numbers, using different diagnostics with varying resolution.

An interesting comparison can be made between the present data at $R e_{0}=\mathbf{5 0 0 0}$ 
and 16000 on figure 11. The increase in Reynolds number causes a broadening of the r.m.s. profile. This effect might be explained by a simple argument concerning timescales near the edge of the jet. Consider parcels of high-concentration fluid that are ejected to the edge of the jet by the turbulent velocity field. As $r$ is increased for a fixed value of $x$, there should be some radial location beyond which smoothing of concentration fluctuations (i.e. mixing) is only weakly influenced by turbulent stirring. In this region, smoothing proceeds at the diffusion rate alone, since the production rate of new interface between fluid constituents is not an important factor. The timescale over which diffusion can act in this outlying region is the timescale associated with re-entrainment by the Biot-Savart induced motions from the jet's largest scales. In a jet flow with a self-similar velocity field, the reentrainment time, $t_{r}$, should be proportional to the local rotation time for a large vortex in the flow (i.e. $t_{\mathrm{r}} \propto D / \bar{U}_{\mathrm{cl}}$ ), and the mean size of the high-concentration parcels, $l_{\mathrm{n}}$, tossed to the edge of the jet should be proportional to $D$ which is proportional to $\left(x-x_{0}\right)$ :

$$
\iota_{\mathrm{r}} \propto \frac{D}{\bar{U}_{\mathrm{cl}}} \propto \frac{\left(x-x_{0}\right)^{2}}{U_{0} d}=\frac{\left(x-x_{0}\right)^{2}}{\nu_{\infty}} R e_{0}^{-1}
$$

An indication of the relative amount of smoothing, due to pure diffusion in a typical high-concentration parcel before re-entrainment, can be obtained from the ratio of the diffusion lengthscale associated with $t_{\mathrm{r}}$, i.e. $\left(D_{\mathrm{j} \infty} t_{\mathrm{r}}\right)^{\frac{1}{2}}$, to the lengthscale of the highconcentration parcel.

$$
\frac{\left(D_{\mathrm{j} \infty} t_{\mathbf{r}}\right)^{\frac{1}{2}}}{l_{\mathrm{h}}} \propto \frac{\left(D_{\mathrm{j} \infty}\left(x-x_{0}\right)^{2} / \nu_{\infty} R e_{0}\right)^{\frac{1}{2}}}{\left(x-x_{0}\right)}=\frac{1}{\left(\operatorname{ScRe} e_{0}\right)^{\frac{1}{2}}} .
$$

An increase in this ratio indicates an increase in the effectiveness of purely diffusive smoothing of the concentration field at the edge of the jet. As indicated by (14), increasing the Reynolds or Schmidt number should decrease the amount of diffusion, and increase the local concentration fluctuation level at the edge of the jet. The proposed effect of $R e_{0}$ can be seen on figure 11 for $r /\left(x-x_{0}\right) \geqslant 0.15$ by comparing the current results at $R e_{0}=5000$ and 16000 . The proposed effect of $S c$ is illustrated on the same figure by the current results at $R e_{0}=5000$ and the measurements of Dahm (1985) $\left(R e_{0}=5000\right.$ and $\left.S c \approx 600-800\right)$. Even though (14) is speculative and not quantitative, note that the result depends only on $d$ and $U_{0}$ in a combination that gives $R e_{0}$.

The region of validity of (14) appears to depend on $R e_{0}$ since the measurements at $R e_{0}=16000$ and 40000 of $C_{\mathrm{rms}}^{\prime}$ are essentially identical (see figures 5 and 9 , recalling that the smooth profile curve for r.m.s. measurements is the same on both figures). Hence, (14) may only be relevant for $R e_{0}$ less than roughly $10^{4}$. Above this value, turbulent stirring may be important all the way to the edge of the jet. Examination of table 2 and figure 11 shows that for $R e_{0}>10^{4}$, higher concentration fluctuations near the edge of the jet are not well correlated with larger experimental values of $\mathrm{ScRe}_{\mathbf{0}}$.

Another comparison worth making is between the current results at $R e_{0}=16000$ and those of Birch et al. (1978) at the same Reynolds number and nearly the same Schmidt number. The mean profiles agree reasonably well but the r.m.s. profiles do not. The disagreement in the r.m.s. profile (see figure 11) might be the result of resolution problems (see discussion below). The mismatch of the centreline r.m.s. levels at the farthest downstream location (see figure 10) might be the result of buoyancy in the experiments of Birch et al. When the buoyancy lengthscale is 
estimated for their experiments, the result is $l_{\mathrm{b}} \approx \mathbf{5 5 d}$, which suggests that their jet may not be momentum dominated beyond $x / d \approx 55$. For comparison, $l_{\mathrm{b}}$ for the present work at $R e_{0}=16000$ is about $440 d$.

Returning to figure 11, it can be seen that the peak values of the r.m.s. profiles do not coincide. While this might be the result of Reynolds-number effects, it might also partially result from resolution difficulties near the centreline. At a given downstream location, the mean passage frequency of concentration scales is greatest at the jet centreline. As the measurement point is moved toward the edge of the jet, the convection velocity decreases, so the bandwidth necessary to capture all of the fluctuations is smaller. If the estimated r.m.s. value of the centreline is low, possible because of insufficient time-space resolution, the rest of the r.m.s. profile is elevated when normalized by the artificially lower value. This centreline resolution difficulty should be most apparent in flows at high Schmidt number because of the smaller size of $\lambda_{\mathrm{B}}$ (see (4)).

\subsection{Power spectra of concentration fluctuations}

The power spectrum of concentration fluctuations, $E_{c}(f)$, was calculated from each of the data sets before optimal filtering using the method described in Appendix $\mathrm{C}$ of Dowling (1988). The overall normalization was chosen such that

$$
\left\{2 \int_{0}^{\infty} E_{c}(f) \mathrm{d} f\right\}^{\frac{1}{2}}=C_{\mathrm{rms}}^{\prime} .
$$

The independent variable is the frequency, $f$ (in $\mathrm{Hz}$ ).

If $E_{c}(f)$ has the same similarity as $\bar{C}$, then it should collapse along rays that emanate from the virtual origin of the jet when scaled by $\bar{C}$ and an appropriate timescale of the jet flow. In this study, the timescale chosen was $\tau_{D}$ (see (11)) which is certainly appropriate for the centreline of the jet flow. Even though a longer timescale should be used for rays off the centreline, $\tau_{D}$ has been used on all of the plots to simplify the presentation of the spectral results. In the far field, the local jet diameter grows linearly with $\left(x-x_{0}\right)$, and the mean centreline velocity decays like $\left(x-x_{0}\right)^{-1}$, so $\tau_{D}$ is proportional to $\left(x-x_{0}\right)^{2}$. Because a single Reynolds number is associated with the far field of the jet, the general mean-flow similarity actually requires that all jet timescales, which are related by a power of the Reynolds number, will have a quadratic dependence on $\left(x-x_{0}\right)$. The passage time of the Kolmogorov scale, for example, increases like $\left(x-x_{0}\right)^{2}$, and is related to $\tau_{D}$ by a factor of $R e_{0}^{-\frac{3}{4}}$.

The figures discussed in this section depict $E_{c}(f)$ on the vertical axis, scaled by the local value of $\tau_{D} \bar{C}^{2}$, and $f$ on the horizontal axis multiplied by $\tau_{D}$. The spectra are plotted in log-log coordinates, so the numerical factors involved in computing the timescale $\tau_{D}$ (the tangent of the jet half-angle $\approx 0.213$, and the constant from Chen \& Rodi's mean centreline velocity decay law $=6.2$ ) only shift the plots relative to the numerical values on the axes and do not affect the spectral shapes. Consequently, other timescales with the same quadratic dependence on the downstream coordinate will produce the same collapse seen in the spectra presented on figures 12-17. All of the spectra in these figures were smoothed with a one-tenth decade filter.

It should be emphasized at the outset of this section that the spectral collapse obtained using $\tau_{D}$, and $C$ does not require any knowledge of either the kinetic-energy dissipation rate or the scalar fluctuation dissipation rate as would be the case for collapsing scalar spectra with Kolmogorov normalization (Gibson 1968). The spectral collapse displayed here is based on the estimates of the largest (and not the smallest) features of the flow. The spectral presentation on figures 12-20 is aimed at 


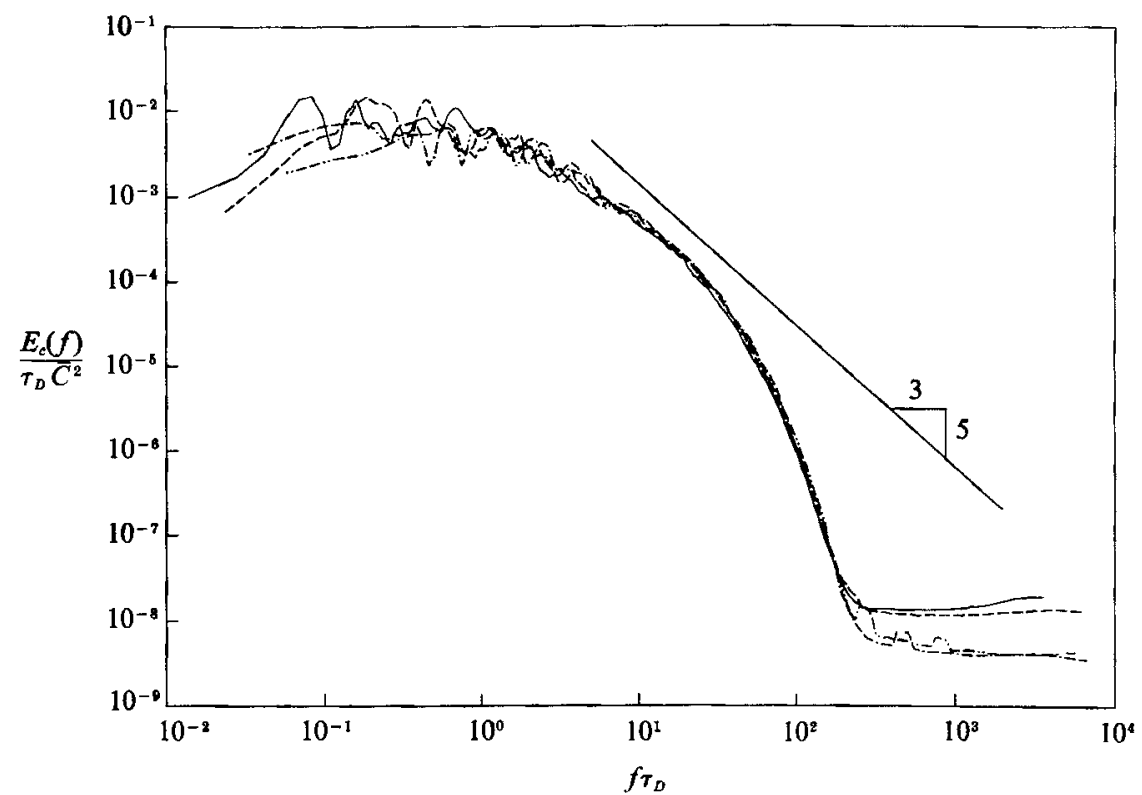

Figure 12. Scaled power spectra of the concentration fluctuations, $E_{c}(f) / \bar{C}^{2} \tau_{D}$, versus dimensionless frequency, $f \tau_{D}$, on the jet centreline at $R e_{0}=5000(--, x / d=20 ;---, 40$; $-\cdot-\cdot-, 60 ;-\cdots-\cdots-, 80$ ).

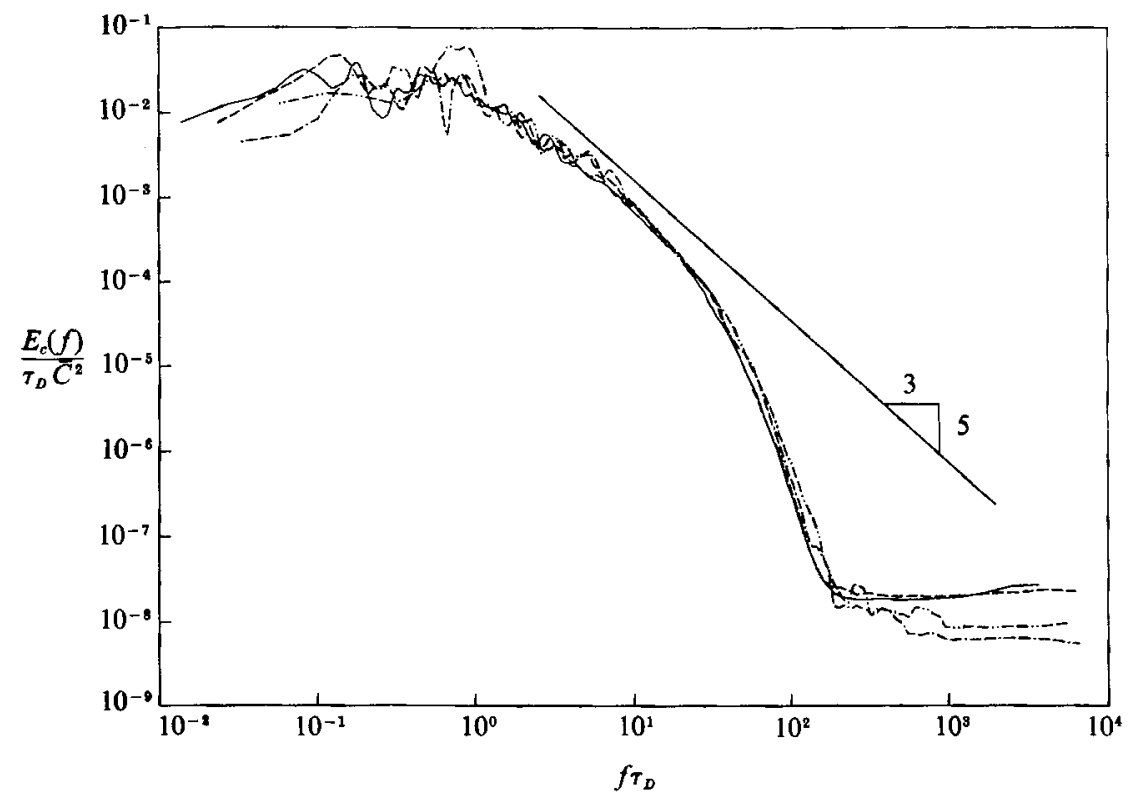

Figure 13. Scaled power spectra of the concentration fluctuations, $E_{c}(f) / C^{2} \tau_{b}$, versus dimensionless frequency, $f \tau_{b}, 3.4^{\circ}$ off the jet centreline at $R e_{0}=5000(-, x / d=20 ;---, 40 ;-\cdot-\cdot-, 60$; $-\cdots-\cdots-, 80$ ).

determining the extent of self-similarity of $E_{c}(f)$ using natural scaling parameters of the turbulent jet. A presentation and discussion of these spectra in Kolmogorovnormalized form is given in Dowling (1988).

Figures 12, 13 and 14 are plots of the scaled values of $E_{c}(f)$ from the measurements 


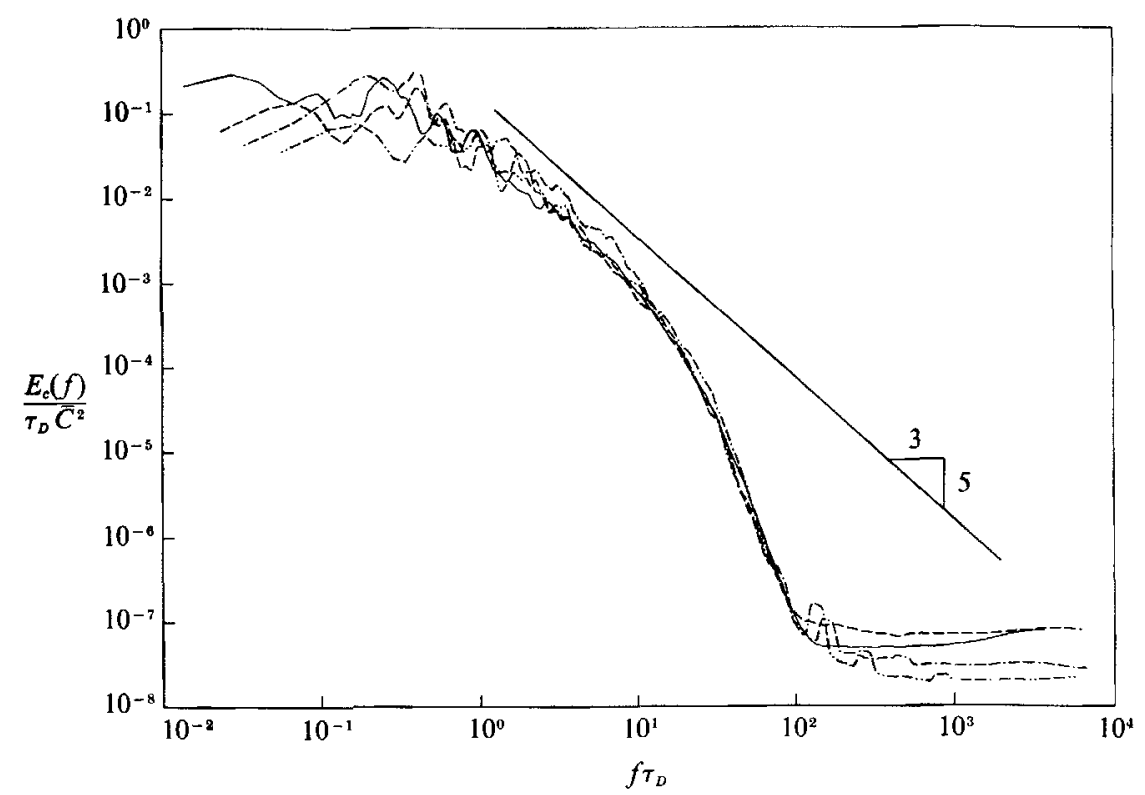

Figure 14. Scaled power spectra of the concentration fluctuations, $E_{c}(f) / \bar{C}^{2} \tau_{b}$, versus dimensionless frequency, $f \tau_{D}, 7^{\circ}$ off the jet centreline at $R e_{0}=5000(-, x / d=20 ;-\cdots, 40 ;-\cdots,-, 60$; $-\cdots-\cdots-, 80)$.

at $R e_{0}=5000$ along the three rays $r /\left(x-x_{0}\right) \approx 0$ (centreline), $r /\left(x-x_{0}\right) \approx 0.06\left(3.4^{\circ}\right.$ off the centreline), and $r /\left(x-x_{0}\right) \approx 0.12\left(7^{\circ}\right.$ off the centreline) for $x / d=20,40,60$, and 80 . The nearly horizontal region of the spectra at high values of $f \tau_{D}$ is the noise floor produced by the measurement technique. The small bumps that occur in the noise floor are the scaled values of $60 \mathrm{~Hz}, 120 \mathrm{~Hz}$, and $360 \mathrm{~Hz}$. The diagnostic system that allowed these spectra to be measured with a dynamic range of nearly seven decades is described in Dowling et al. (1989).

The spectra collapse well in spite of the modest Reynolds number of the flow. Deviations from a perfect collapse, which are more evident as the edge of the jet is approached, are probably attributable to decreased statistical convergence. In particular, for a fixed run time at a given downstream location, the total effective sample size is smaller near the edge of the jet, where the mean velocity is lower, the turbulence is intermittent, and there is less mixed fluid to sample. Other reasons for the deviations could be small variations in $R e_{0}$ from run to run, and minor angular differences $\left( \pm 0.2^{\circ}\right)$ between the actual measurement locations and exact alignment along a given ray. The quality of the collapse displayed on figures 12, 13 and 14 for $E_{c}(f)$, and on figure 8 for $C_{\mathrm{rms}}^{\prime}$ is strong verification that $C_{\mathrm{rms}}^{\prime}$ and $\bar{C}$ follow the same specific similarity law throughout the jet. Recall that $E_{c}(f)$ and $C_{\mathrm{rms}}^{\prime}$ are related through (15), so figure 8 is an integral measure of the specific similarity of $E_{c}(f)$ at $R e_{0}=5000$.

The adequacy of the sampling rate of the measurements is evident through the length of the portion of each measured spectra that is devoted to the noise floor. Clearly, any further increase in the sampling rate for these runs would not have 'uncovered' any more of the turbulent concentration signal spectra. The adequacy of the spatial resolution can also be ascertained from the spectra. These experiments were designed to resolve a calculated value of $\lambda_{B}((4)$, with $\beta=1)$, At $R e_{0}=5000$, the calculated value of $f_{\mathrm{B}} \tau_{D}$ is about 670 . If there were any effect on the measurements 
from the finite spatial resolution of the detection system, it would be manifest in the spectra for $f_{\mathrm{B}} \tau_{D}$ near and above 670 . Because this region of the spectra falls below the noise floor for all of the plotted results, the portion of the measured spectra at frequencies below $f_{\mathrm{B}} \tau_{D} \approx 670$, and above the noise floor can be regarded as free of any resolution difficulties. More importantly, however, the collapse of the spectra at different axial stations at high frequency supports the claim of no resolution difficulties.

For locally isotropic turbulent flow at high Reynolds number with Schmidt number of order unity, the spectrum of turbulent scalar fluctuations is expected to display a $-\frac{5}{3}$ power-law region (Batchelor 1959; Gibson 1968; Monin \& Yaglom 1975). The Kolmogorov (Batchelor) lengthscale passage frequency should correspond to the high-frequency end of this region when a proper value of $\beta$ is used in (4) to compute $\lambda_{\mathrm{K}}$. Previous spectral investigations by Clay (1973), Chapmann (1979) and others put the actual value of $\beta$ at little more than 10 . While the spectra plotted on figures 12-14 are clearly not from a high-Reynolds-number flow, the scaled frequency range in which they begin to fall more rapidly than a constant power law is close to the value of $f_{\mathrm{B}} \tau_{D}(\approx 54)$ calculated with $\beta=12.5$. This figure for $\beta$ was suggested by Professor Carl Friehe (personal correspondence, 1988). Clay and Friehe worked on a heated, and perhaps slightly buoyant, jet flow at a Reynolds number of almost $10^{6}$. On figures $12-14$, the choice of the same timescale for each ray causes the numerical location of the spectral break point (if one can be considered to exist on these plots) to decrease with increasing $r /\left(x-x_{0}\right)$.

At the low-frequency end, $f \tau_{D} \leqslant 1$, the spectra on figures $12-14$ are approximately flat or show a mild peak in the range $0.1 \leqslant f \tau_{D} \leqslant 1.0$. While this behaviour is consistent with the passage of structures whose scale is approximately the same as the local diameter, the most general conclusion to be drawn is that the fluctuations that occur at frequencies below $1 / \tau_{D}$ do not have a greater amplitude than those that occur near $1 / \tau_{D}$. Consequently, one can think of the turbulent cascade at this $R e_{0}$ as starting at a lengthscale which is approximately the size of the local jet diameter.

Although the spectra collapse along rays when scaled by $\tau_{D}$ and $\bar{C}$, the spectra are different from ray to ray in ways that cannot be corrected by changing the timescale used to non-dimensionalize the axes of the plots. In particular, the spectra along the ray at $7^{\circ}$ show a longer power-law region with a slope closer to $-\frac{5}{3}$ than those from the inner rays. This latter behaviour is also apparent in the spectra reported by Lockwood \& Moneib (1980) at $x / d=20$ in a heated air jet at $R e_{0} \approx 50000$.

For the data recorded at $R e_{0}=16000$, the resolution requirements were relaxed by a factor of three. This choice was made because it put the value of $\frac{1}{3} f_{\mathrm{B}} \tau_{D}(\approx 1600 / 3$ with $\beta=1$ ) right at the point where the signal contribution to the measured spectra met the noise floor, thereby making the best use of the available experimental bandwidth. Figures 15,16 and 17 are plots of the scaled values of $E_{c}(f)$ from the measurements at $R e_{0}=16000$ along the three rays $r /\left(x-x_{0}\right) \approx 0$ (centreline), 0.06 $\left(3.4^{\circ}\right.$ off the centreline) and $0.11\left(6.3^{\circ}\right.$ off the centreline) for $x / d=30$ and 90 . The slopes of the diagonal lines on these figures do not have a theoretical basis and were drawn only to aid the eye. The quality of the collapse shown on figures 15-17 implies that all of the conclusions from the lower Reynolds number concerning the specific similarity of $C_{\mathrm{rms}}^{\prime}$ and $E_{c}(f)$ remain valid at $R e_{0}=16000$. As before, incomplete statistical convergence, the intermittent character if the flow near the edge of the jet, small differences in $R e_{0}$, and imperfect angular alignment may well be responsible for the small deviations from a perfect collapse.

These spectra show the same general shape as those at $R e_{0}=5000$ except for the 


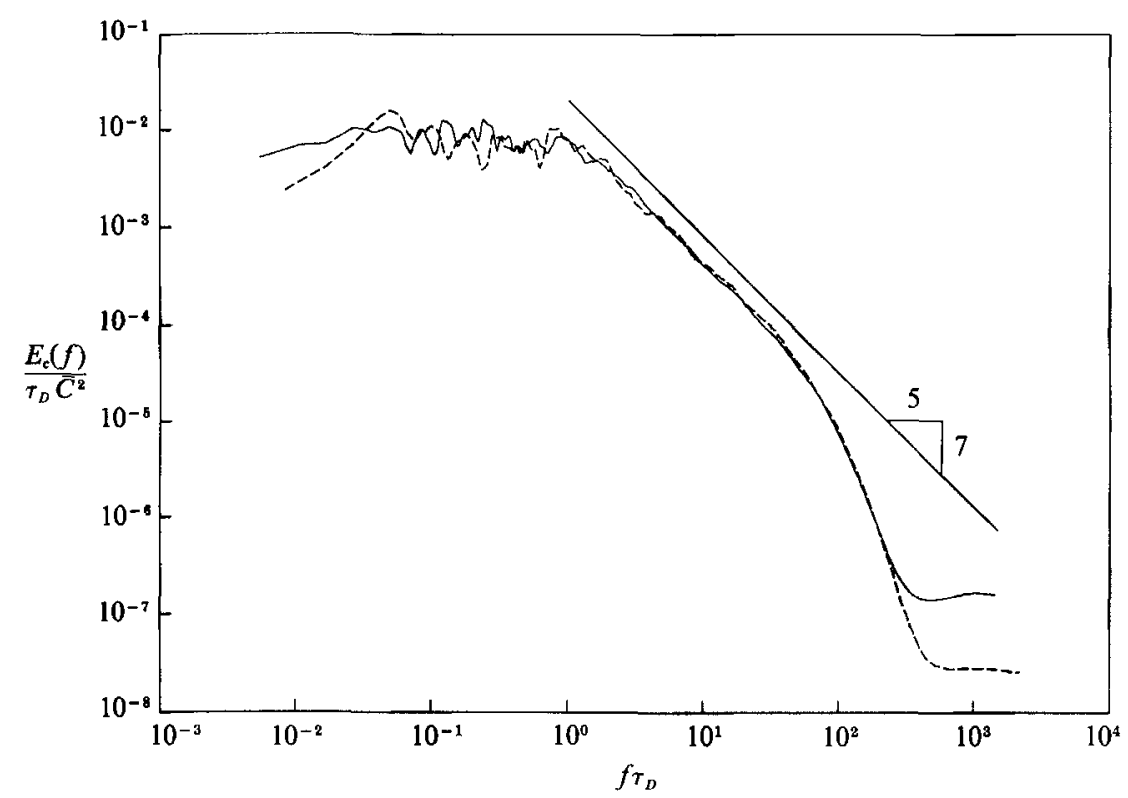

Figure 15. Scaled power spectra of the concentration fluctuations, $E_{c}(f) / \bar{C}^{2} \tau_{D}$, versus dimensionless frequency, $f \tau_{D}$, on the jet centreline at $R e_{0}=16000(-, x / d=30 ;-\cdots, 90)$.

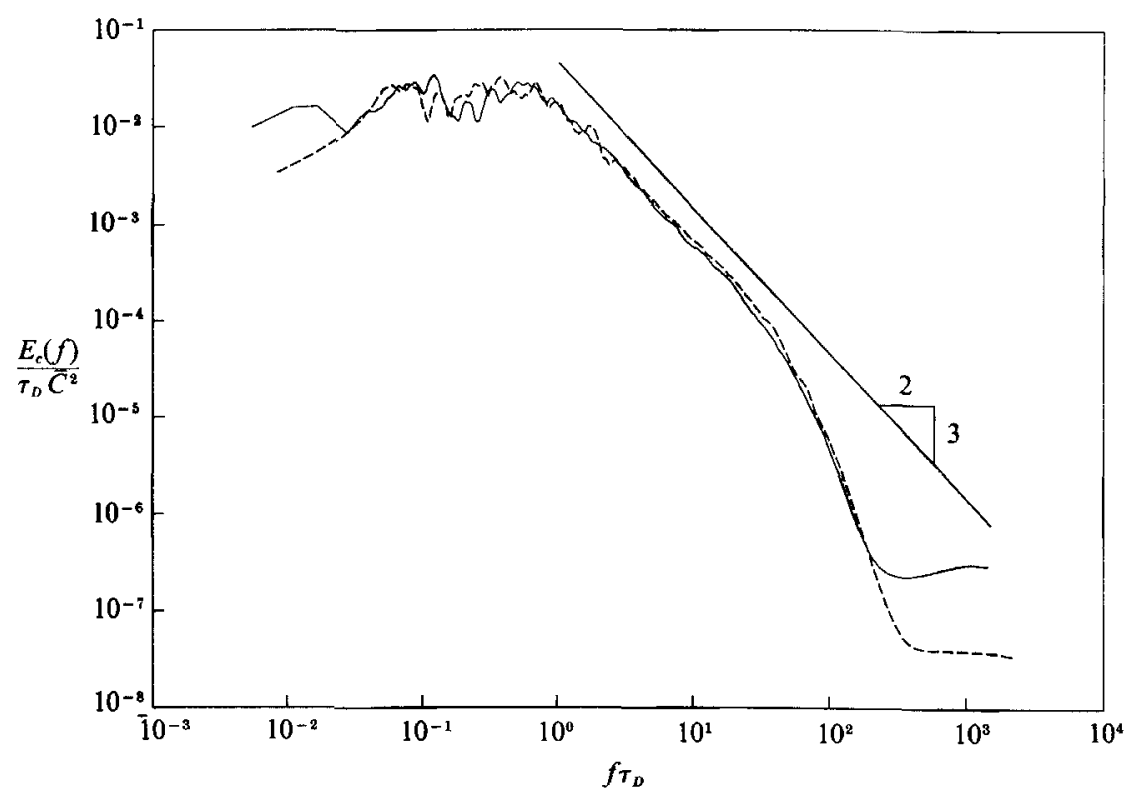

FIGURE 16. Scaled power spectra of the concentration fluctuations, $E_{c}(f) / \bar{C}^{2} \tau_{D}$, versus dimensionless frequency, $f \tau_{D}, 3.4^{\circ}$ off the jet centreline at $R e_{0}=16000(-, x / d=30 ;---,, 90)$.

appearance of longer power-law regions, which extend the high-frequency range of the spectra. The slope of the power-law region steepens as the edge of the jet is approached. The spectra from the centreline appear have a slope of about -1.4 , which decreases to -1.5 on the ray $3.4^{\circ}$ off the centreline, while the straightest part of the spectra from the ray $6.3^{\circ}$ off the centreline has a slope close to $-\frac{5}{3}$. The low- 


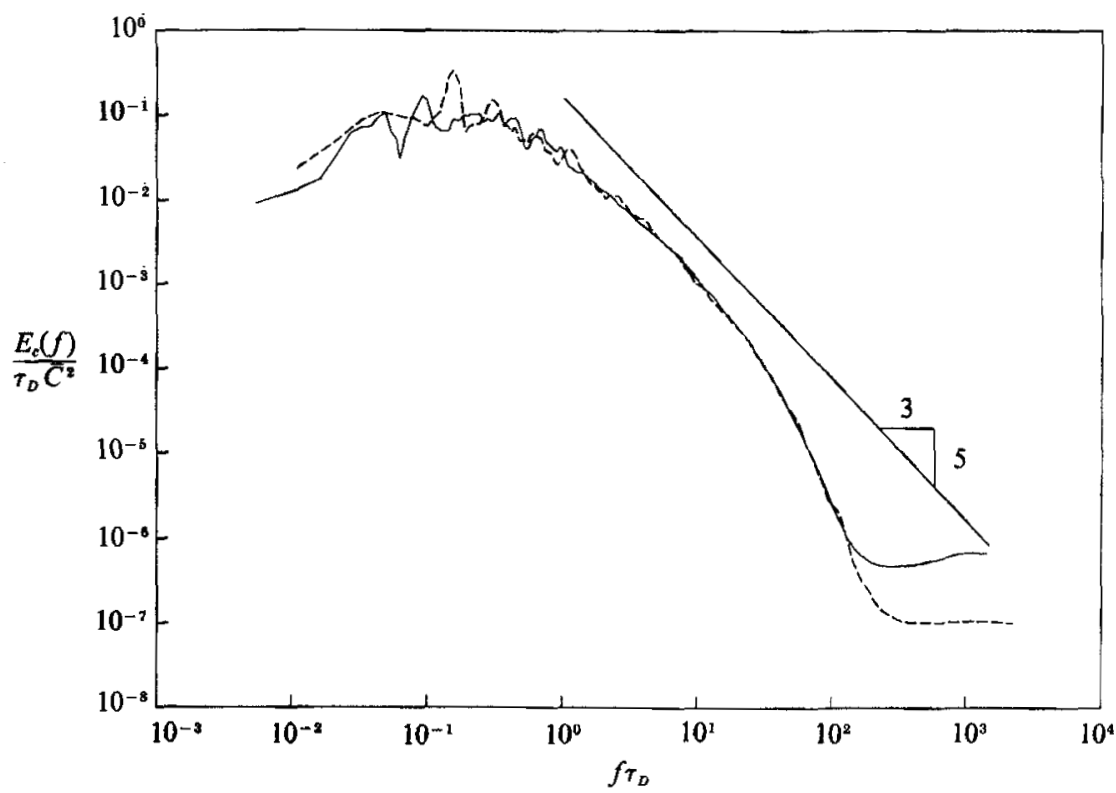

FiquRE 17. Scaled power spectra of the concentration fluctuations, $E_{c}(f) / \bar{C}^{2} \tau_{D}$, versus dimensionless frequency, $f \tau_{D}, 6.3^{\circ}$ off the jet centreline at $R e_{\theta}=16000(-, x / d=30 ;-\cdots,-90)$.

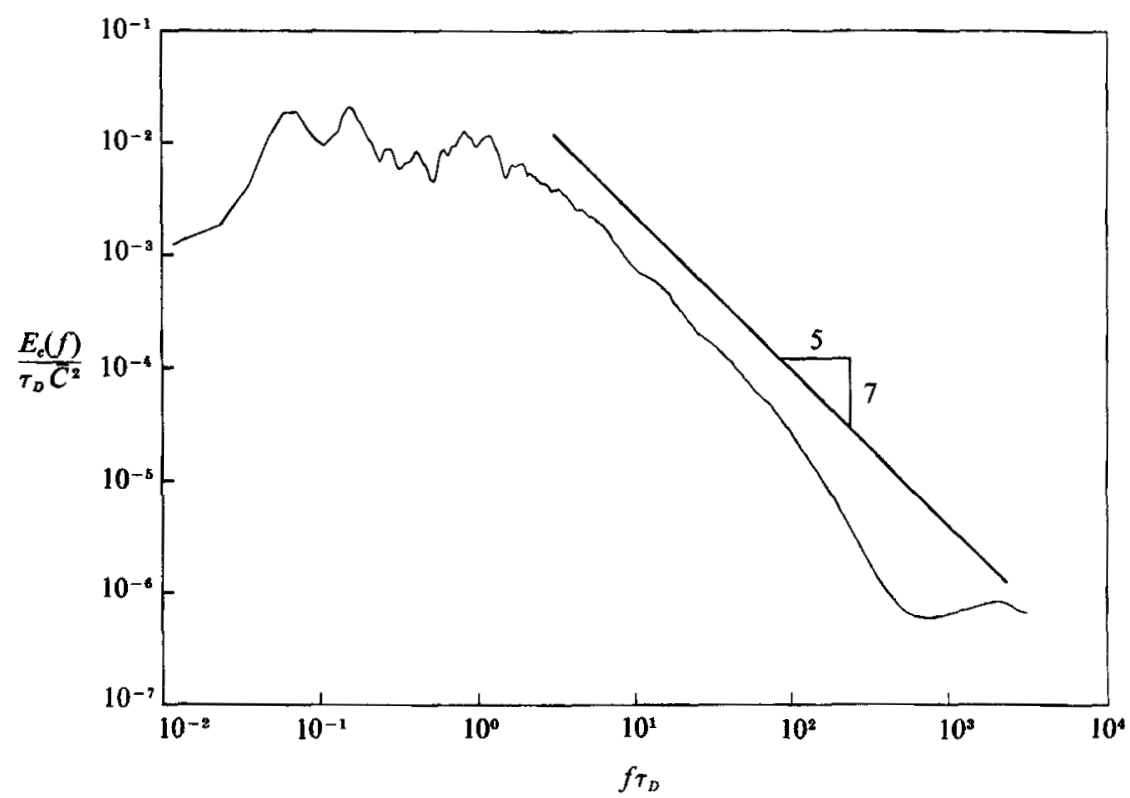

Froure 18. Scaled power spectra of the concentration fluctuations, $E_{c}(f) / \bar{C}^{2} \tau_{D}$, versus dimensionless frequency, $f \tau_{D}$, on the jet centreline at $R e_{0}=40000$ and $x / d=60$.

frequency ends of the spectra at $R e_{0}=16000$ have the same behaviour as the spectra at $R e_{0}=5000$.

Figures 18 and 19 depict $E_{c}(f)$ at $R e_{0}=40000, x / d=60$ and $r /\left(x-x_{0}\right) \approx 0$ and 0.12 . The axis scaling is the same as before except that here $x_{0}$ was taken to be zero. In both cases, the full, high-frequency behaviour of the jet is partially masked by the measurement noise. The power-law regions are slightly longer than those at 


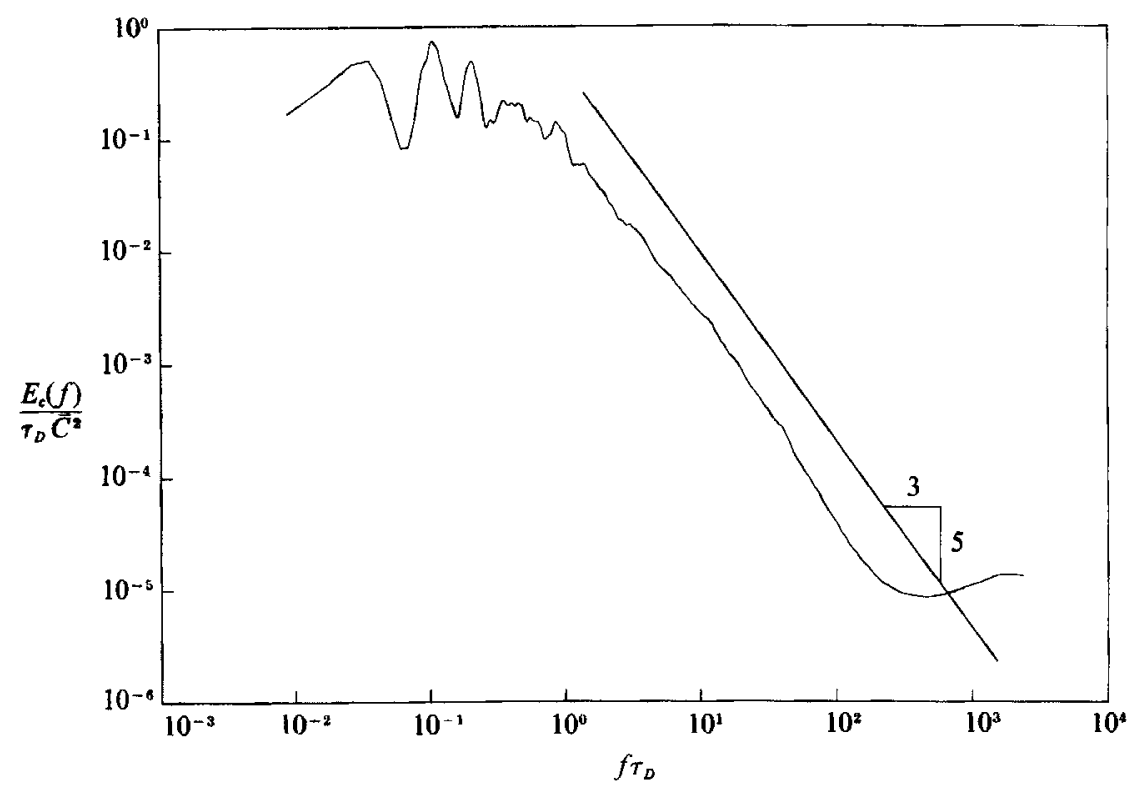

Fiaure 19. Scaled power spectra of the concentration fluctuations, $E_{c}(f) / \bar{C}^{2} \tau_{D}$, versus dimensionless frequency, $f \tau_{D}, 7^{\circ}$ off the jet centreline at $R e_{0}=40000$ and $x / d=60$.

$R e_{0}=16000$, and the slopes are consistent with those displayed by the same rays at the lower Reynolds numbers. It is possible that the observed spatial variation of exponents for the measured spectra are not peculiar to the Reynolds-number range investigated here, and might persist at higher Reynolds numbers. Therefore, the exponent of the spectral power-law region along a particular ray might be a general similarity property of the jet concentration field. The low-frequency ends of the spectra on figures 18 and 19 have the same behaviour as that of the spectra at the smaller Reynolds numbers.

The physical reasons for the differences in power-law-region exponents, and their deviation from $-\frac{5}{3}$, are unknown. Yakhot, She \& Orszag (1989) argue that the inertialrange velocity spectra of homogeneous turbulence might produce spectral power-law exponents that differ from $-\frac{5}{3}$, so perhaps the turbulent scalar field might also produce spectra with power-law exponents that differ from $-\frac{5}{3}$. The spectral differences found seem to indicate that jet turbulence is statistically different from ray to ray.

A comparison of previously reported scalar spectra from the jet centreline with the current results is given on figure 20 . The parameters necessary to scale the spectra from other experiments and make them satisfy the overall normalization (equation (15)) were taken from the author's papers in all but one case. The vertical location of the spectrum of Clay (1973) could not be determined because he did not include $C_{\mathrm{rms}}^{\prime}$ among his results. Its vertical location on figure 20 was chosen to match that of the spectrum of Becker et al. (1967), whose Reynolds number of 54000 was elosest to that of Clay.

The current results and those of Becker et al. (1967) agree quite well for $f \tau_{D}$ less than unity. This implies that the concentration fluctuation energy associated with the largest scales is independent of Reynolds number for $R e_{0} \geqslant 5000$. The centreline spectrum of Lockwood \& Moneib (1980) probably falls below the others in this frequency range because their reported centreline r.m.s. level, at the downstream location of their spectral measurement $(x / d=20)$, is low $\left(C_{\mathrm{rms}}^{\prime}=0.16 \bar{C}\right)$ when 


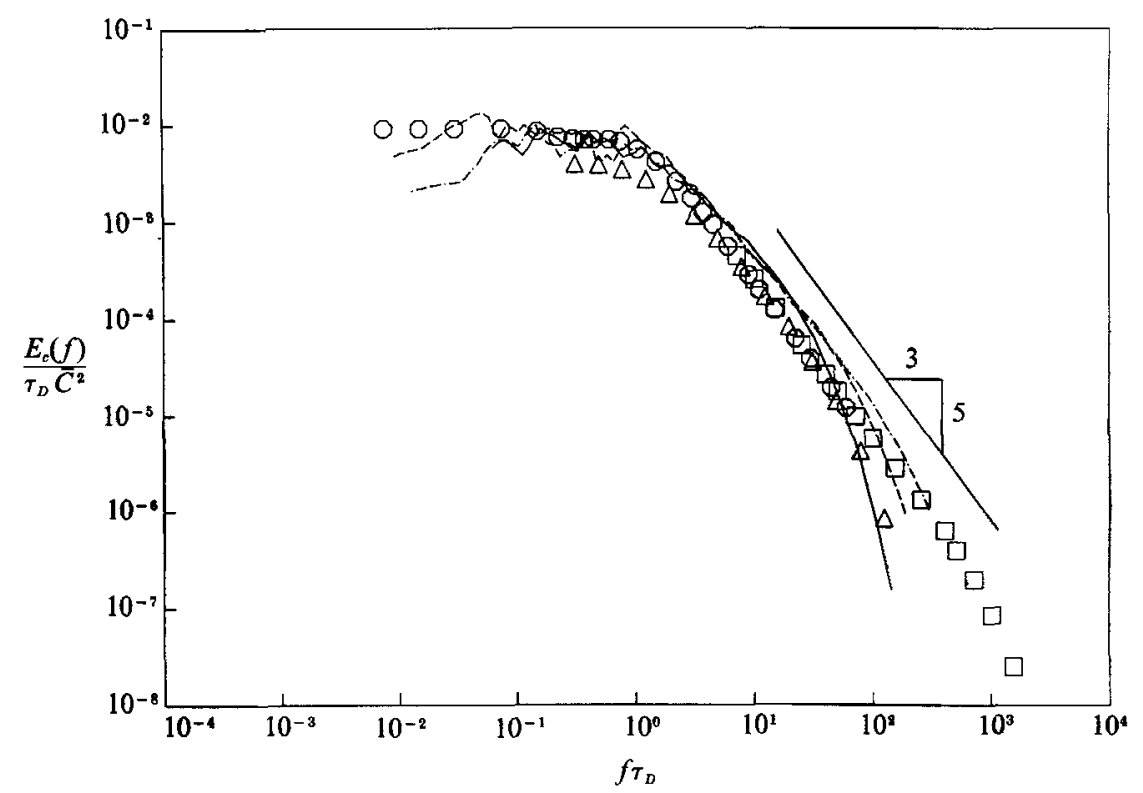

Figure 20. Comparison of the current results to previous experiments for the scaled centreline concentration fluctuation power spectra, $E_{c}(f) / \overline{C^{2}} \tau_{D}$, versus dimensionless frequency, $f \tau_{D}(\square$, Clay 1973; $\bigcirc$, Becker et al. 1967; $\triangle$, Lockwood \& Moneib 1980; present results,$- R e_{0}=5000$; ,$\left.--- R e_{0}=16000 ;-\cdot--, R e_{0}=40000\right)$. Reynolds and Schmidt number information for the plotted data is available in table 2 .

compared to that of the other experiments. Hence, the behaviour of the largest concentration scales is likely to be a general similarity property of the jet. This contention has some backing, at present, from the liquid-phase (i.e. Schmidt number $\approx 600$ ) measurements of Weddell (1953) and Dahm, Dimotakis \& Broadwell (1984). They showed that the flame length in reacting jet flows becomes independent of Reynolds number above $R e_{0} \approx 3000$. Because the largest scales of motion dominate the entrainment process in shear layers and jets (Brown \& Roshko 1974; Dimotakis, Miake-Lye \& Papantoniou 1983; Dahm \& Dimotakis 1987), the Reynolds-number independence of the largest concentration scales is consistent with these flame-length observations.

The centreline spectra of Becker et al. (1967) and Clay (1973) closely follow a $-\frac{5}{3}$ power law over a significant range, whereas the current centreline results do not. This might result from the influence of buoyancy in the Clay's heated jet which discharged horizontally. Buoyancy may have deflected the effective centreline of the jet from the geometrical centreline, thereby altering the jet's character, and complicating the proper geometrical placement of the measurement probe with respect to the nozzle exit. The reasons for the difference in the spectral exponent between the current centreline spectrum at $R e_{0}=40000$ and that of Becker et al. are unknown. Note also that the spectrum of Lockwood \& Moneib (1980) does not entirely coincide with that of Becker et al. either, even though the Reynolds numbers of these two studies were very close. However, if the centreline spectrum of Lockwood \& Moneib is raised so that its level is the same as that of the other spectra at $f \tau_{D} \approx 1$, it would be a close match with the current spectra at $R e_{0}=16000$ and 40000 .

These differences do not appear to have a simple explanation. From the currently accepted theoretical standpoint (Batchelor 1959; Gibson 1968; see also Monin \& 
Yaglom 1975), it is unlikely that the differences in Schmidt (Prandtl) number between experiments could have any effect on the spectral content of the scalar field at frequencies below the passage frequency of the Kolmogorov scale, which is of order $10^{2}$ or greater on the scaled abscissa for all the experimental results shown on figure 20 . It also seems unlikely that there would be a significant Reynolds-number effect confined to the small range between 40000 (the highest of the current study) and 54000 (the Reynolds number of Becker et al.). It would appear that the source of these spectral differences must lie in experimental differences between these investigations which effect the character of the jet's concentration field either directly, or through the turbulent velocity field which convects the measured scalars.

Regardless of the exact shape or levels, one Reynolds-number effect can be seen in figure 20. This is the movement to higher frequency, with increasing Reynolds number, of the break point at which a spectrum starts to fall faster than a constant power law. This observation was, of course, anticipated since $f_{\mathrm{B}} \tau_{D} \propto \lambda_{\mathrm{K}}^{-1} \propto R e^{\frac{3}{7}}$. The spectrum from Lockwood \& Moneib was computed from data that were filtered at frequencies higher than $f \tau_{D} \approx 50$, and therefore breaks from the constant-power-law behaviour at a lower frequency than expected. Taken all together, this observation, the above discussion, and figures 12-20, lead to the conclusion that the exact shape of the jet's concentration fluctuation power spectrum is specifically self-similar, but not generally self-similar.

\subsection{Probability density function of concentration}

After each data set was optimally filtered, a histogram of the instantaneous concentration divided by the local mean concentration, $C / \bar{C}$, was compiled by sorting the data into bins. This histogram was normalized; i.e.

$$
\int_{0}^{\infty} \operatorname{PDF}(C / \bar{C}) \mathrm{d}(C / \bar{C})=1,
$$

to form a probability density, $\operatorname{PDF}(C / \bar{C})$. The independent variable, $C / C$, was chosen because dividing $C$ by $\bar{C}$ should remove the effects of the downstream decay of the mean concentration. For fixed $R e_{0}$, the probability density function of $C / \bar{C}$ should depend only on $r /\left(x-x_{0}\right)$ if the statistical properties (higher moments) of the distribution of concentration fluctuations in the jet follow the same specific similarity law as the first moment, $\bar{C}$. Additionally, the PDFs presented below are plotted with a linear abscissa and a linear ordinate to determine the extent to which they can be considered self-similar. Logarithmic axes or additional self-normalization with anything besides $\bar{C}$ would only obscure the main conclusion to be drawn from this section: the PDF of $C / \bar{C}$ is self-similar along rays that emanate from the jet's virtual origin.

Figures 21, 22 and 23 display the measured PDFs of $C / \bar{C}$ along the three rays at $r /\left(x-x_{0}\right) \approx 0,0.06$ and 0.12 for $x / d=20,40,60$ and 80 at $R e_{0}=5000$. The quality of the collapse of the distributions along rays implies that $C / \bar{C}$ is the proper specific similarity variable, and that the higher moments of the PDF of $C / \bar{C}$ are independent of the downstream position in the jet. For example, the square root of the second moment of the PDF of $C / \bar{C}$, taken about $C / \bar{C}=1$, is $C_{\mathrm{rms}}^{\prime} / \bar{C}$, which was previously found to depend only on $r /\left(x-x_{0}\right)$ for fixed $R e_{0}$ (see $\left.\$ 3.1\right)$. Slight imperfections in the collapse are believed to be the consequence of incomplete statistical convergence, the intermittent character of the flow at the larger values of $r /\left(x-x_{0}\right)$, and possibly 


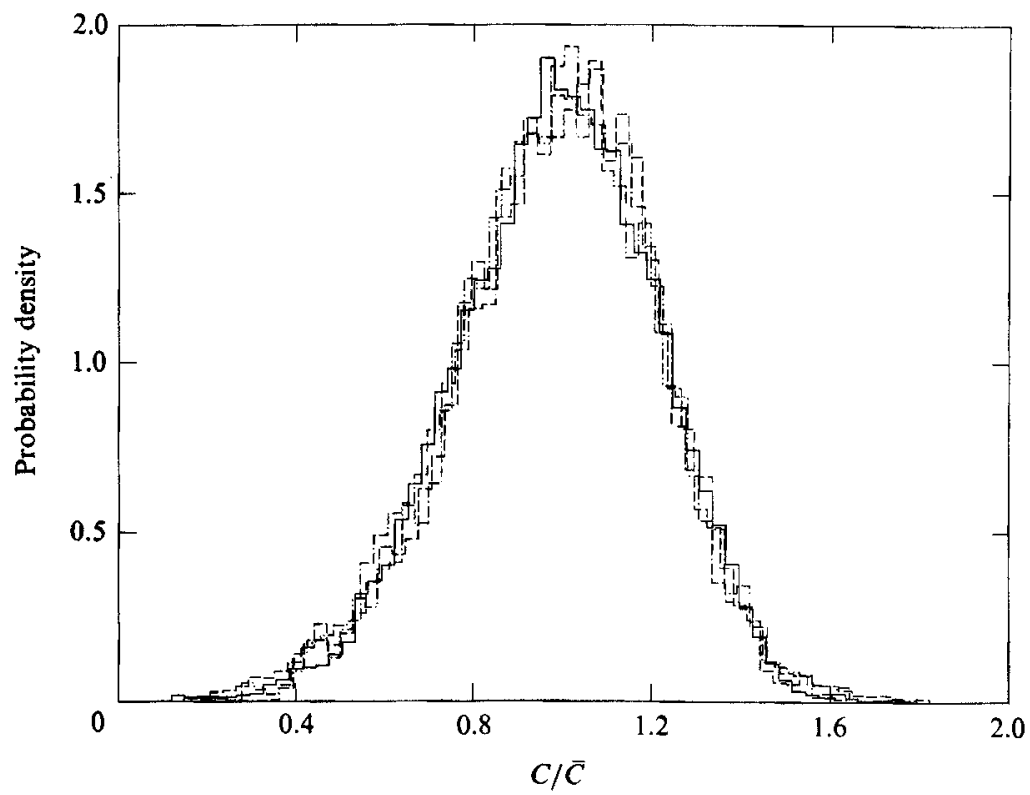

Figure 21. Probability density function of the scaled concentration, $C / \bar{C}$, on the jet centreline at $R e_{0}=5000(-, x / d=20 ;----, 40 ;-\cdot-\cdot, 60 ;-\cdots-\cdots-, 80)$.

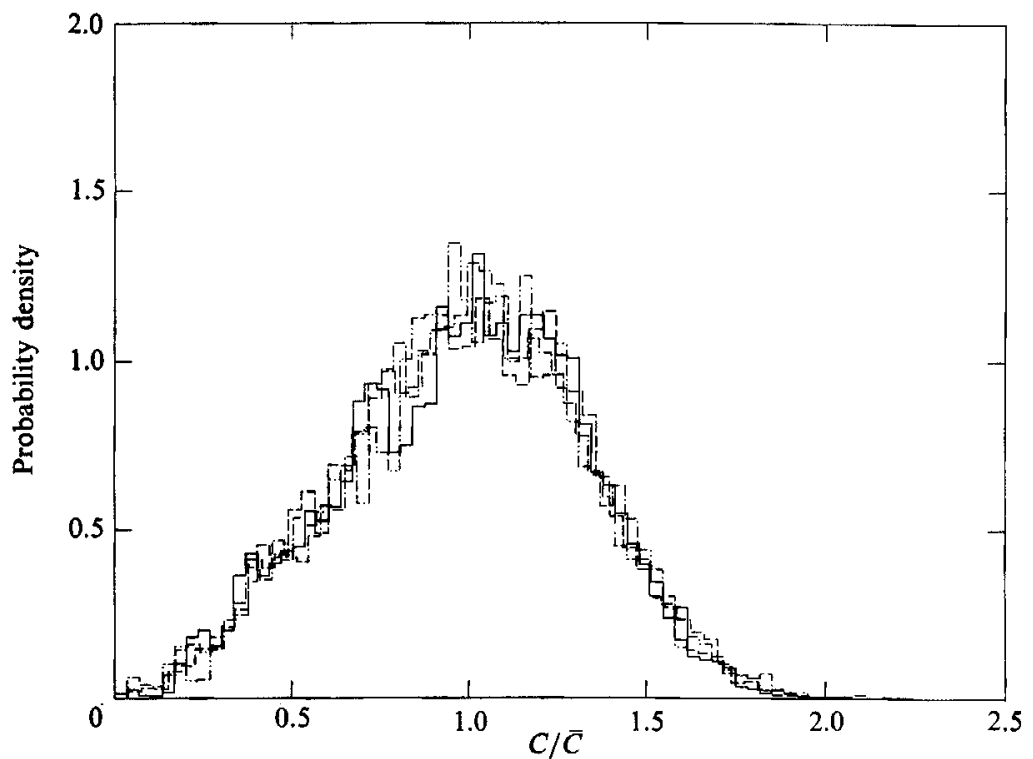

FIgure 22. Probability density function of the scaled concentration, $C / \bar{C}, 3.4^{\circ}$ off the jet centreline at $R e_{0}=5000(-, x / d=20 ;---, 40 ;-\cdots \cdot-60 ;-\cdots, \cdots, 80)$.

imperfect alignment of individual measurement points with respect to the chosen ray (note the difference between the PDFs at $\eta=0.11$ and $\eta=0.12$ on figure 26).

Figures 24, 25 and 26 depict the measured probability density functions of $C / \bar{C}$ along the three rays at $r /\left(x-x_{0}\right) \approx 0,0.06$ and 0.11 to 0.12 , for $x / d=30$ and 90 , at $R e_{0}=16000$. The quality of the collapse is good, implying that the proposed specific 


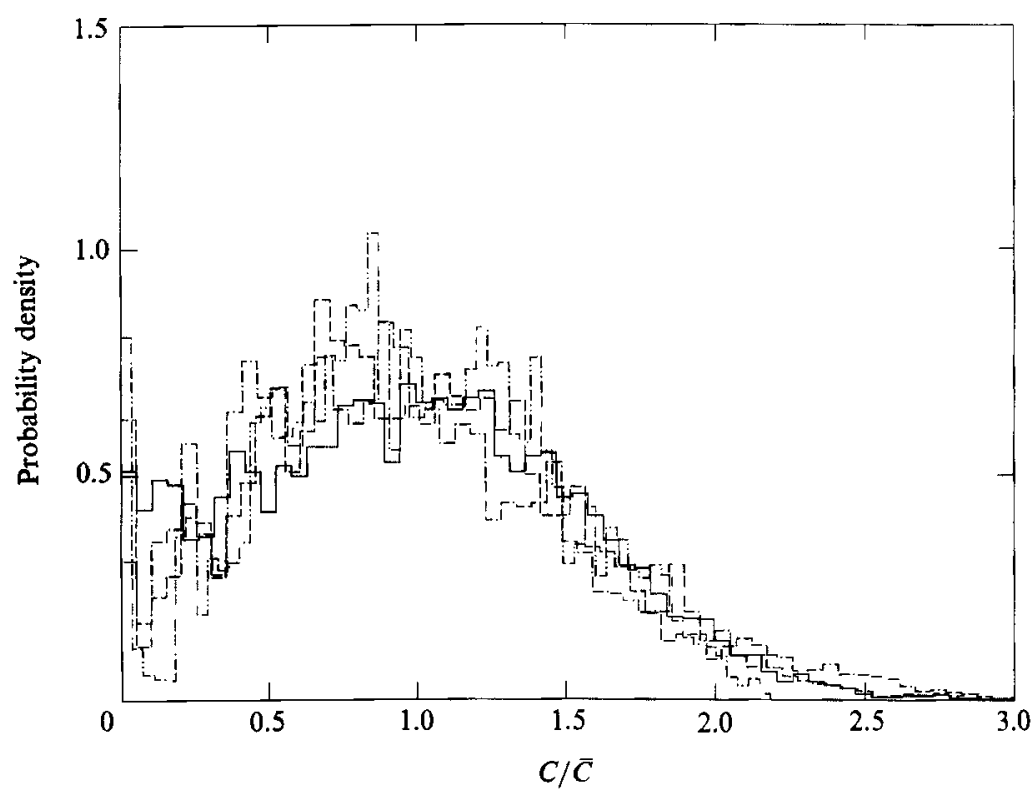

Figure 23. Probability density function of the scaled concentration, $C / \bar{C}, 7^{\circ}$ off the jet centreline at $R e_{0}=5000(-, x / d=20 ;----, 40 ; \cdots \cdot-, 60 ; \cdots-\cdots, 80)$.

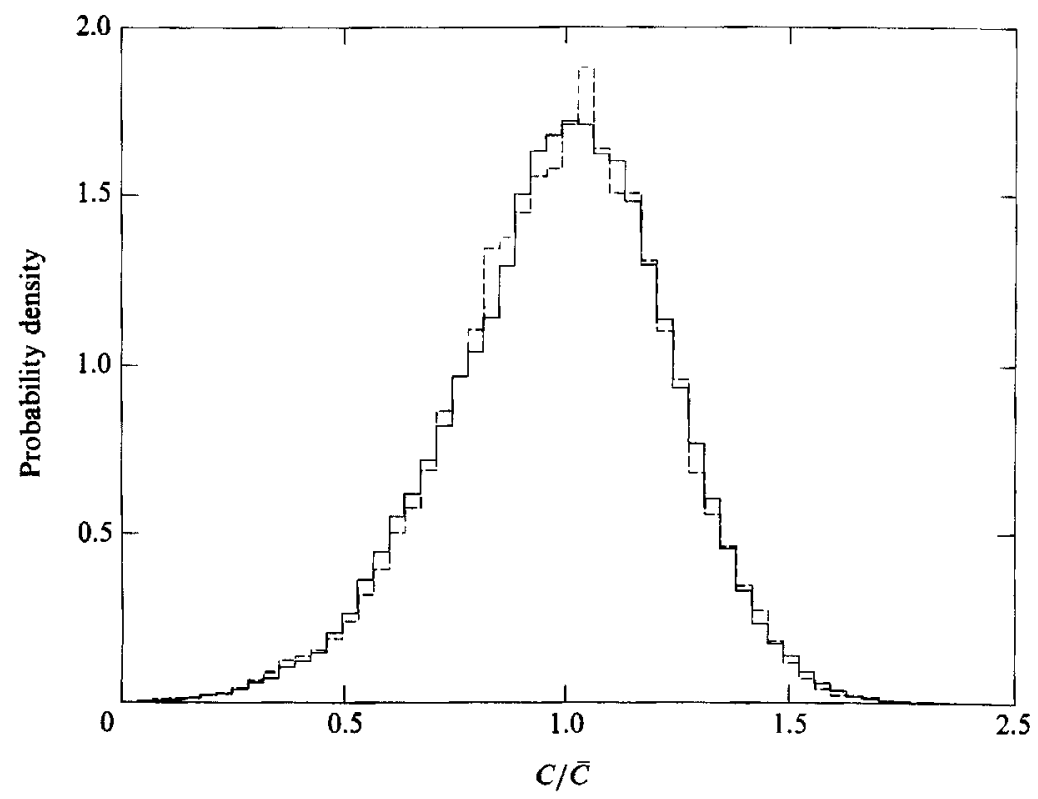

Figure 24. Probability density function of the scaled concentration, $C / \bar{C}$, on the jet centreline at $\operatorname{Re}_{0}=16000(-, x / d=30 ;-\cdots, 90)$.

similarity variable, $C / \bar{C}$, for the PDF of concentration fluctuations is a general similarity variable, even though the actual shapes of the PDFs of $C / \bar{C}$ might depend on $R e_{0}$. Imperfections in the collapse at this Reynolds number may be attributable to the same problems mentioned in the previous paragraph.

The area contained in the first bin $(C \approx 0)$ of the plotted PDFs corresponds to unmixed fluid from the reservoir. Its contribution to the whole PDF must increase 


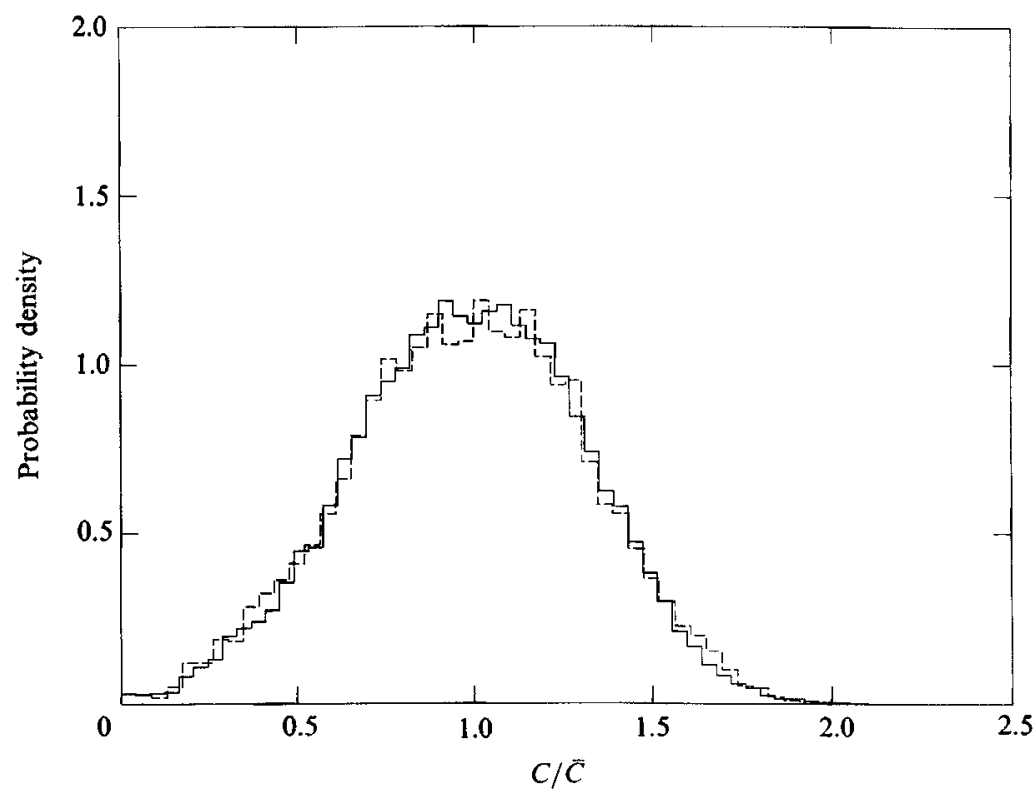

Froure 25. Probability density function of the scaled concentration, $C / \bar{C}, 3.4^{\circ}$ off the jet centreline at $R e_{0}=16000(-, x / d=30 ;----, 90)$.

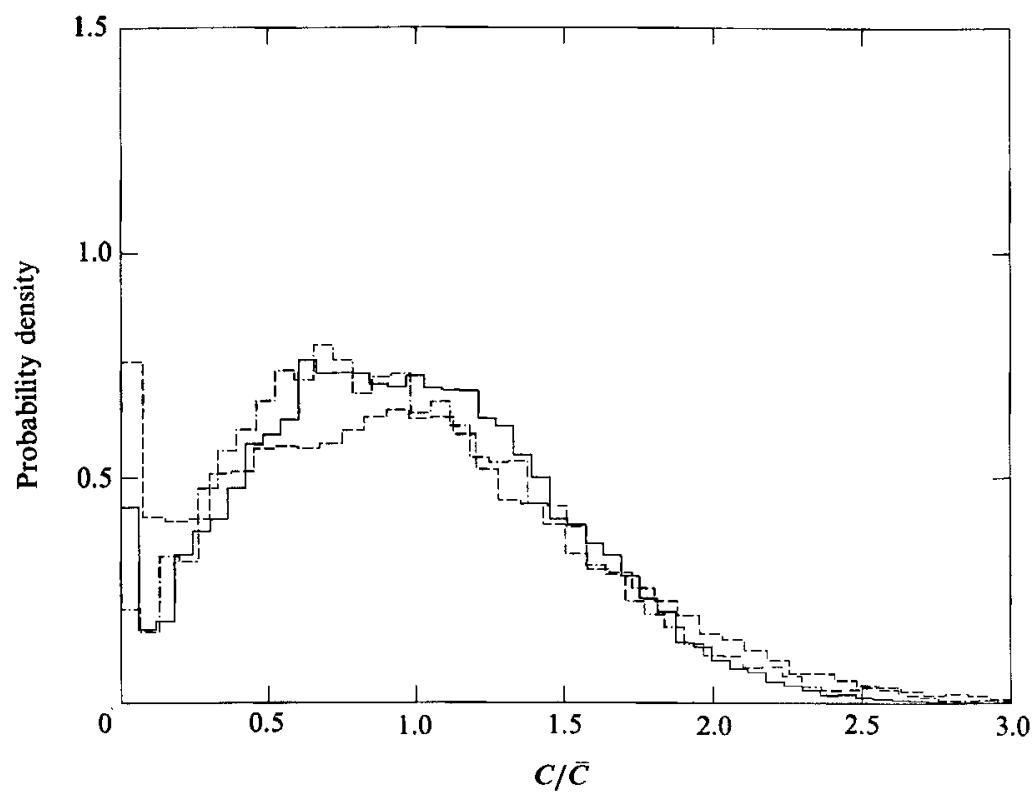

Fiqure 26. Probability density function of the scaled concentration, $C / \bar{C}, 6.3^{\circ}$ to $7^{\circ}$ off the jet centreline at $R e_{0}=16000(-, x / d=30, \eta \approx 0.11 ;---, x / d=30, \eta \approx 0.12 ; \cdots \cdot-\cdot, x / d=90$, $\eta \approx 0.11$ ).

as $r /\left(x-x_{0}\right)$ increases until the measurement point is completely outside the jet, and the probability of finding reservoir fluid is unity. Therefore, the area of the first bin of the PDF can be taken as a measure of one minus the intermittency of the jet's concentration field, where the intermittency is understood to be the fraction of the time that the concentration field at a point can be considered turbulent. Figures 23 
and 26 appear to show that one minus the intermittency, by this measure, varies by as much as factor of three. While this could certainly result from a sampling interval that was too short in comparison with the timescales of the flow, the basis for the above intermittency observation is put in further doubt when the finite precision of the experimental calibration is accounted for. Unlike the bulk of the PDF, the absolute accuracy of the calibration (about \pm 1 part per $10^{3}$ ) is an important limit in the interpretation of concentration measurements near $C=0$. For example, the mean concentration value of the run at $x / d=90$ that was used to create figure 26 was about 0.025 , which means that each plotted PDF bin covers a range of only 0.0005 (roughly half the calibration error). Additionally, because it is possible to find smooth regions of the jet's concentration field near its edge with $C \neq 0$, the distinction between turbulent and non-turbulent fluid should perhaps be based on a threshold for $|\mathrm{d} C / \mathrm{d} t|$ and not just a threshold on $C / \bar{C}$. To conclude, the limitations of the finite sampling interval, the calibration accuracy, and the lack of a proper turbulent/nonturbulent distinction criterion prevent the inclusion of any quantitative statements concerning the intermittency inferred from the reported PDFs of $C / \bar{C}$. Chatwin \& Sullivan (1989) discuss the shortcomings of the standard definition of the intermittency for a dispersing scalar in turbulent flow.

At this point, one direct Schmidt-number comparison can be made with the results of Dahm (1985) at $R e_{0}=5000$ and $S c \approx 600-800$. He found pure reservoir fluid on the centreline of the jet at least $3 \%$ of the time. In the current study at $R e_{0}=5000$, pure reservoir fluid was not found on the jet centreline (see figure 21 near $C=0$ ). While this difference could result from calibration inaccuracy, which makes the $C=0$ level of the data uncertain, the size of the observed discrepancy between $S c=1.0$ and $S c \approx 600-800$ is larger than that which can be produced from the current calibration uncertainty alone. Hence, the reservoir fluid's lateral turbulent diffusivity appears to have an 'inverted' dependence on the molecular diffusivity, because pure reservoir fluid reaches the jet centreline at $S c \approx 600-800\left(D_{\mathrm{j} \infty} \ll \nu_{\infty}\right)$, but not at $S c=1.0\left(D_{\mathrm{j} \infty}\right.$ $=v_{\infty}$ ). This inverse behaviour of the effective diffusivity of a scalar in the presence of both molecular and convective transport has also been noted by Taylor (1953) for flow through a tube.

Figure 27 is a comparison of the averaged and smoothed centreline PDFs of $C / \bar{C}$ at $R e_{0}=5000,16000$ and 40000. The PDFs for $R e_{0}=5000$ and 16000 plotted on this figure were obtained by combining those on figures 21 and 24 , respectively. The distribution for $R e_{0}=16000$ is a little shorter and a little wider than the others. This is consistent with the slightly higher value of $C_{\mathrm{rms}}^{\prime} / \bar{C}$ on the centreline at this Reynolds number (see figure 10). The differences in the three distributions on figure 27 are small, which implies that the PDF of $C / \bar{C}$ on figure 27 is a good candidate for a general centreline similarity PDF.

This contention is supported, for the most part, by a comparison of this research with other investigations (see figure 28 ). The bulk of the measurements, including all of the gas-phase results, collapse well. Two investigations in liquid-phase jets at high Schmidt number (Dahm 1985, axial measurements; and Papantoniou 1985; both in dark symbols on figure 28), produce PDFs that are significantly broader than the rest of the investigations. It is unlikely that these two PDFs differ from the rest because of Schmidt-number effects since other high-Schmidt-number efforts (Dahm 1985, radial measurements; and Papanicolaou \& List 1988) agree with the lower Schmidt and Prandtl-number results. It is also unlikely that the observed differences are the result of Reynolds-number effects since the shape of the centreline PDF of $C / \bar{C}$ from investigations at Reynolds numbers from 3000 (Papanicolaou \& List 1988) to 52000 


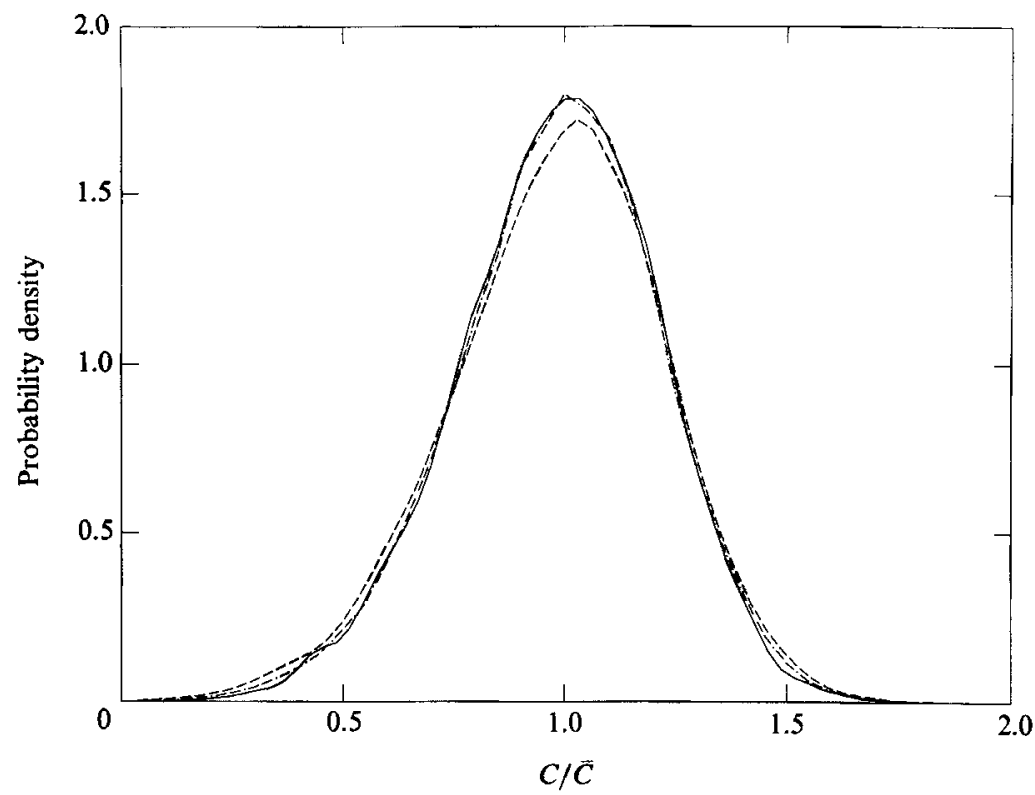

Figure 27. Averaged probability density functions of the scaled concentrations, $C / \bar{C}$, on the jet centreline $\left(-, \operatorname{Re}_{0}=5000 ;----, 16000 ;-\cdot-\cdot-40000\right)$.

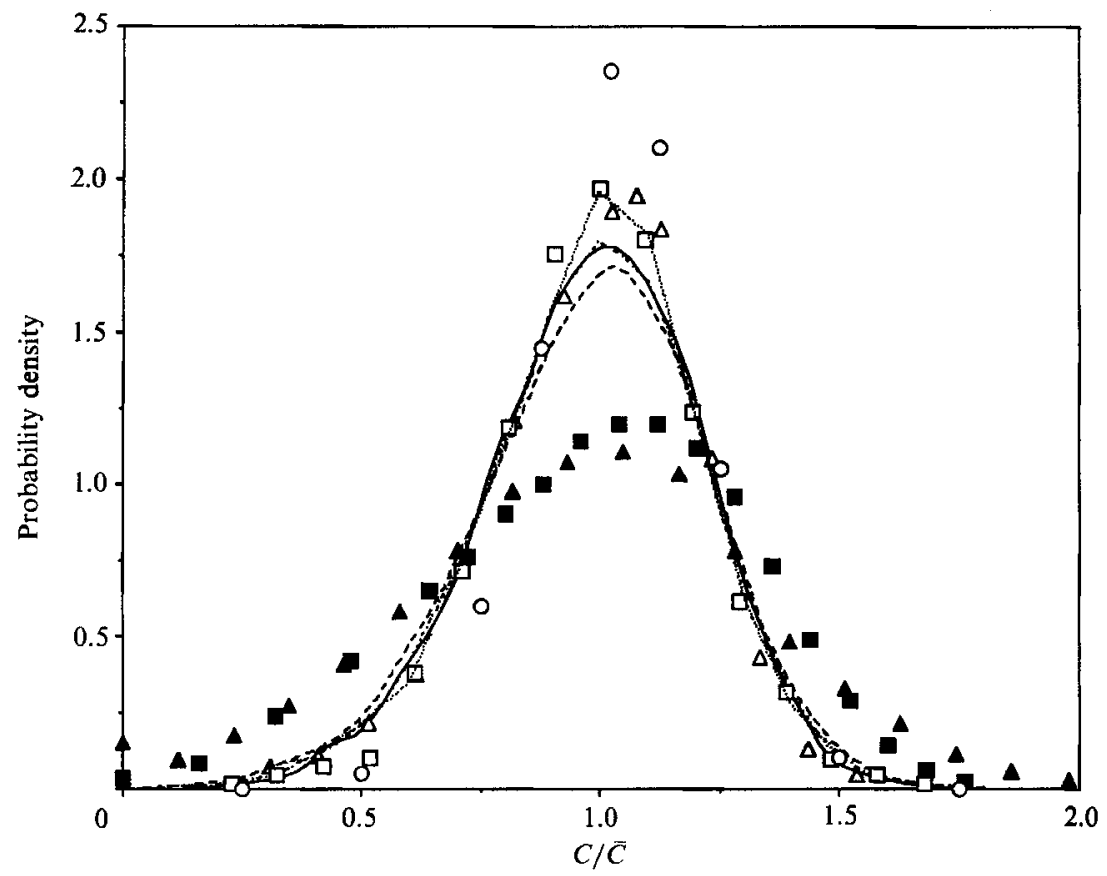

Figure 28. Comparison of reported centreline probability density functions of concentration with the concentration axis scaled by the local mean concentration $(\triangle$, Dahm 1985, radial measurements; $\square$, Dahm 1985, axial measurements; $\square$, Lockwood \& Moneib 1980; Papantoniou 1985; O, Papanicolaou \& List 1987; $\cdots \cdots$, Papanicolaou \& List 1988; current results at $R e_{0}=5000 ;---$, current results at $R e_{0}=16000 ; . .-$, current results at $R e_{0}=40000$ ). Reynolds and Schmidt number information for the plotted data is available in table 2 . 


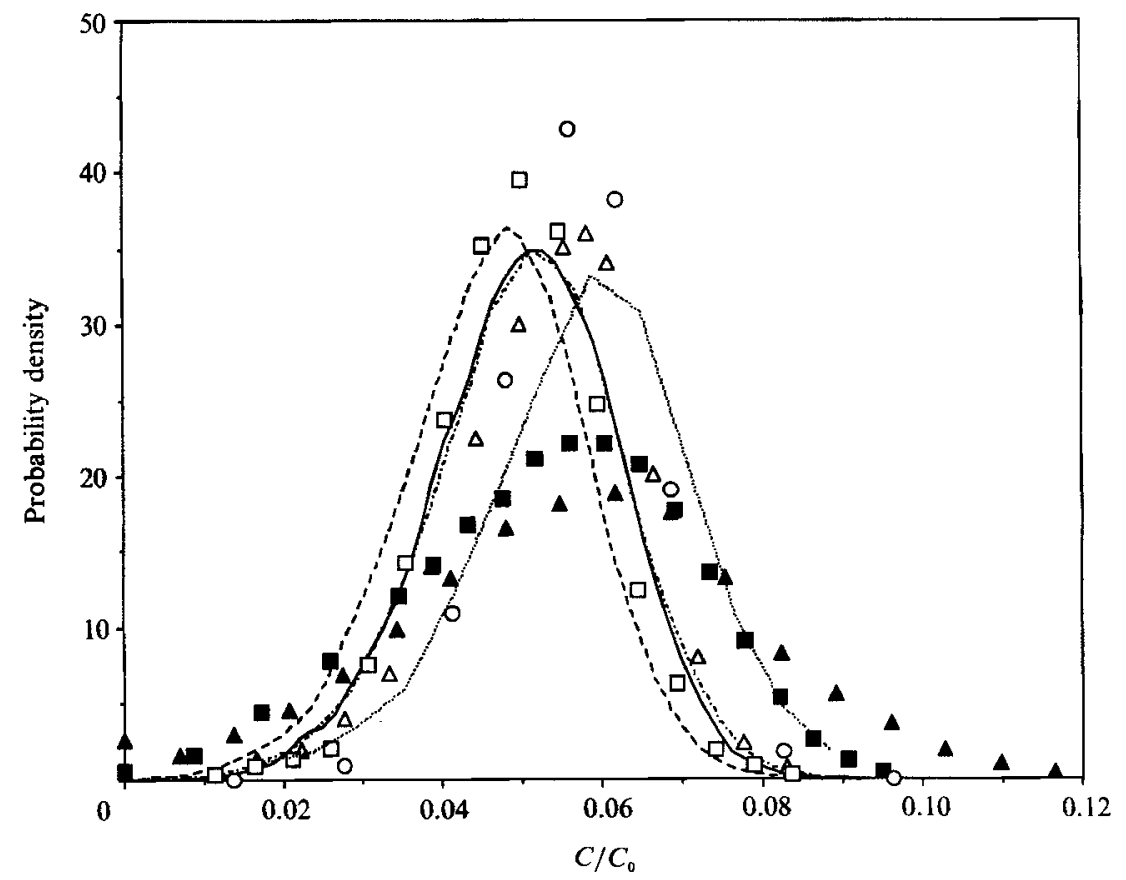

FiguRE 29. Comparison of reported centreline probability density functions of concentration without scaling the concentration axis by the local mean concentration ( $\triangle$, Dahm 1985, radial measurements; $\square$, Dahm 1985, axial measurements; $\square$ Lockwood \& Moneib 1980; $\Delta$, Papantoniou 1985; O, Papanicolaou \& List 1987; $\cdots \cdots$. Papanicolaou \& List 1988; current results at $R e_{0}=5000 ;----$, current results at $R e_{0}=16000 ;-\cdot-\cdot-\cdot$, current results at $R e_{0}=40000$ ). $C_{0}$ is the jet exit concentration. Reynolds and Schmidt number information for the plotted data is available in table 2. All PDFs are referenced to $\chi=100$.

(Lockwood \& Moneib 1980) is essentially the same. The difference in the two groupings on figure 28 is probably too large to be caused by resolution differences between experiments. However, resolution differences could result in the comparatively mild scatter in the 'preferred' PDF-shape grouping (open symbols and dashed/dotted lines on figure 28 ).

The scaling of the abscissa in figures 21-28 hides a major problem in the understanding of jet similarity : there is no precise and accepted value for $\kappa$ (the mean centreline concentration decay parameter, see \$3.1). The demonstrated general similarity variable of the jet's concentration field, $C / \bar{C}$, removes any dependence on the value of $\kappa$. Figures 28 and 29 illustrate this point. Both figures depict the same data. The difference is the abscissa of figure 29 , which is the absolule relative concentration, $C / C_{0}$, for a particular downstream position $\left(C_{0}\right.$ is the jet exit concentration). All of the PDFs on figure 29 have been referenced to $\left(x-x_{0}\right) / d^{*}=100$ for the purposes of comparison, using the mean centreline concentration decay law from each experiment, and the assumption that the PDF of $C / \bar{C}$ was specifically selfsimilar in each experiment. The difference in the quality of the collapse between figure 28 and 29 is entirely the result of the variation in $\kappa$ (see (1) and figure 7).

Another important point that should be emphasized is that figures 27 and 28 are for the centreline only. Since the r.m.s. profile of concentration fluctuations may depend on Reynolds number and/or Schmidt number near the edge of the jet (see discussion in $\S 3.1$ ), the PDF of $C / \bar{C}$ may also. In fact, the evidence of figure 11 for 
$R e_{0} \approx 10^{4}$ suggests that the width of the PDF of $C / \bar{C}$ on a given ray near the edge of the jet $\left(r /\left(x-x_{0}\right)>0.15\right)$ should increase with increasing $R e_{0}$ or $S c$. Hence, while it appears that the PDF of $C / \bar{C}$ is generally self-similar near the centreline, and perhaps out as far as $r /\left(x-x_{0}\right) \approx 0.12$ (compare figures 22 with 25 , and 23 with 26 ), PDFs of $C / \bar{C}$ significantly off the jet centreline may only be specifically self-similar along rays.

A final matter that should be discussed involves the statistical coupling of the PDF of $C / \bar{C}$ and $E_{c}(f)$ through the r.m.s. concentration fluctuation, $C_{\mathrm{rms}}^{\prime}$, i.e.

$$
\bar{C}^{2} \int_{0}^{\infty}(C / \bar{C}-1)^{2} \operatorname{PDF}(C / \bar{C}) \mathrm{d}(C / \bar{C})=2 \int_{0}^{\infty} E_{c}(f) \mathrm{d} f=C_{\mathrm{rms}}^{\prime 2}
$$

This relation suggests that $C_{\mathrm{rms}}^{\prime}$ on the centreline, and therefore the centreline PDF of $C / \bar{C}$, should depend on $R e_{0}$ since the length of the power-law region of $E_{c}(f)$ is found to depend on $R e_{0}$ (see final paragraph of \$3.2). While this is mathematically correct, we note that as the Reynolds number is raised above $R e_{0} \approx 5000$, the integrated contribution from the extended portion of $E_{c}(f)$ is a small fraction of the total r.m.s. fluctuation level, an amount perhaps too small to detect in the background of other experimental uncertainties in the present Reynolds-number range from 5000 to 40000. The largest contributions to the r.m.s. fluctuation level comes from the spectral region near $f \tau_{D} \approx 1$, which has been found to be independent of $R e_{0}$ above $R e_{0}=5000$.

\section{Conclusions}

This work leads to several conclusions. For a fixed Reynolds number and Schmidt number, the jet's turbulent concentration field is not only self-similar along rays in the mean, but is statistically self-similar along rays at every time- and lengthscale as demonstrated by the collapse of the power spectrum of concentration fluctuations. This detailed similarity along rays appears to be a general similarity property of the jet because it was found at two values of $R e_{0}(5000$ and 16000). One set of general similarity parameters for the turbulent jet's concentration field appears to be $\bar{C}$ and $\tau_{D}$ (or any lengthscale that has a quadratic dependence on downstream distance). Use of these should effect a collapse of the statistical measures of the fluctuating concentration field in the jet at any turbulent Reynolds number. The shape of the PDF of $C / \bar{C}$ near the centreline in gas-phase jets appears to be self-similar in general (except for the small caveat mentioned at the end of $\$ 3.3$ ), which implies that $C_{\mathrm{rms}}^{\prime} / \bar{C}$ has a general self-similar value on the centreline between 0.23 and 0.24 . Results to date suggest that the PDF of $C / \bar{C}$ may only be specifically self-similar away from the jet centreline. And finally, the contribution of the largest concentration scales to the strength of the concentration fluctuations in the jet is generally self-similar above $R e_{0}=5000$.

This work also points out the clear need for further experimentation to determine the factors that control the decay parameter, $\kappa$. Such an investigation will likely require extensive measurements of the jet's velocity field, which regrettably were beyond the scope of this study.

The authors wish to acknowledge useful discussions with Dr J. E. Broadwell, and thank Professor W. A. Dahm for providing the digitized values of the 'pre-1986' experimental results for figures $6,7,10$, and 11. These experiments would not have been possible without the electronic wizardry of Dr Dan Lang. Thanks also to Paul Miller for his insightful suggestions, and proof reading of the text. This work was 
supported by the Gas Research Institute under grants 5083-260-0878 and 5087-2601467, and the Air Force Office of Scientific Research under grants 83-0213 and 88-0155.

\section{REFERENCES}

Avery. J. F. \& Faeth, G. M. 1974 Combustion of a submerged gaseous oxidizer jet in liquid metal. Fifteenth Intl Symp. on Combustion, pp. 501-512. The Combustion Institute, Pittsburgh, Pennsylvania.

BAtcheloR, G. K. 1959 Small scale variation of convected quantities like temperature in turbulent fluid. J. Fluid Mech. 5, 113-139.

Becker, H. A., Hottel, H. C. \& Williams, G. C. 1967 The nozzle fluid concentration field of the round turbulent jet. J. Fluid Mech. 30, 285-303.

Birch, A. D., Brown, D. R., Dodson, M. D. \& Thomas, J. R. 1978 The turbulent concentration field of a methane jet. J. Fluid Mech. 88, 431-449.

Brown, G. L. \& Roshko, A. 1974 On density effects and large structure in turbulent mixing layers. J. Fluid Mech. 64, 775-816.

Chapmann, D. R. 1979 Computational aerodynamics development and outlook. AIAA J. 17, p. $1293-1313$.

Chatwin, P. C. \& Sullivan, P.J. 1989 The intermittency factor of scalars in turbulence. Phys. Fluids A 1, 761-763.

Chen, C. J. \& Rodi, W. 1980 Vertical Turbulent Buoyant Jets: A Review of the Experimental Data. Pergamon.

ClAy, J.P. 1973 Turbulent mixing of temperature in air, water, and mercury. Ph.D. thesis, University of California San Diego, La Jolla Ca.

Corrsin, S. \& Uberoi, M. S. 1950 Further experiments on the flow and heat transfer in a heated turbulent air jet. NACA Rep. 998.

DАнм, W. A. 1985 Experiments on entrainment, mixing, and chemical reactions in turbulent jets at high Schmidt number. Ph.D. thesis, California Institute of Technology, Pasadena, CA.

DAнm, W. A. \& Dimotakis, P. E. 1987 Measurements of entrainment and mixing in turbulent jets. AIAA J. 25, 1216-1223.

Dahm, W. A., Dimotakis, P. E. \& Broadwell, J. E. 1984 Non-premixed turbulent jet flames. AIAA paper 84-0369.

Dimotakis, P. E., Miake-Lye, R. C. \& Papantoniou, D. A. 1983 Structure and dynamics of round turbulent jets. Phys. Fluids 26, 3185-3192.

Dowling, D. R. 1988 Mixing in gas phase turbulent jets. Ph.D. thesis, California Institute of Technology, Pasadena CA.

Dowling, D. R. \& Dimotakis, P. E. 1988 On mixing and structure of concentration field of turbulent jets. In Proc. First National Congress on Fluid Dynamics, 25-28 July 1988 Cincinnati, Ohio, Part 2, pp. 982-988. AIAA.

Dowling, D. R., Lang, D. B. \& Dimotakis, P. E. 1989 An improved laser-Rayleigh scattering photodetection system. Exp. Fluids 7, 435-440.

Fischer, H. G., LIst, E. J., Кон, R. C. Y., IмBerger, J. \& Brooks, N. H. 1979 Mixing in Inland and Coastal Waters. Academic.

Friehe, C. A., Van Atta, C. W. \& Gibson, C. H. 1971 Jet turbulence: dissipation rate measurements and correlations. AGARD Turbulent Shear Flows, CP-93, pp. 18-1 to 18-7.

GEORGE, W. K. 1989 The self-preservation of turbulent flows and its relation to initial conditions and coherent structures. In Advances in Turbulence (ed. W. K. George \& R. Arndt), pp. 39-73. Hemisphere.

Girson, C. H. 1968 Fine structure of scalar fields mixed by turbulence. Il Spectral theory. Phys. Fluids 11, 2316-2317.

Hivze, J. O. 1975 Turbulence, 2nd edn., pp, 224-225, 520-523. McGraw-Hill.

KUEThe, A. M. 1935 Investigations of the turbulent mixing regions formed by jets. Trans. ASME E: J. Appl. Mech. 1, 87-95. 
Landat, L. D. \& Lifshitz, E. M. 1959 Fluid Mechanics, Pergamon.

Lockwood, F.C. \& MoNwiB, H. A. 1980 Fluctuating temperature measurements in a heated round free jet. Combust. Sci. Tech. 22, 63-81.

Monin, A. S. \& Yaglom, A. M. 1975 Statistical Fluid Mechanics, vol. II, Chap. 8. MIT Press.

Niwa, C., Ichizawa, J., Yoshikawa, N. \& Ohtake, K. 1984 Time-resolved concentration measurements of jets by laser Rayleigh method - comparison of $\mathrm{He}, \mathrm{CO}_{2}$, and $\mathrm{CCl}_{2} \mathrm{~F}_{2}$ jets. Proc. Fourteenth Intl Symp. on Space Technology and Science, Tokyo.

Papanicolaou, P. N. \& List, E. J. 1987 Statistical and spectral properties of the tracer concentration in round buoyant jets. Intl J. Heat Mass Transfer 30, 2059-2071.

PapanicolaOU, P. N. \& List, E. J. 1988 Investigations of round turbulent buoyant jets. J. Fluid Mech. 195, 341-391,

Papantoniou, D. 1985 Observations in turbulent buoyant jets by use of laser-induced fluorescence. Ph.D. thesis, California Institute of Technology, Pasadena, Ca.

PiTTs, W. M. 1986 Effects of global density difference and Reynolds number variations on mixing in turbulent axisymmetric jets. Natl Bur. Stand. Internal Rep. 86-3340.

Press, W. H., Flannery, B. P., Teukolsky, S. A. \& Vetterling, W. T. 1986 Numerical Recipes, pp. 417-419. Cambridge University Press.

REICHARDT, H. 1965 Turbulenten Strahlausbreitung in Gleichgerichteter Grundstromung. Forsh. Ing.-Wes. 30, 133-139; topic developed in: Zur problematik der turbulenten Strahlausbreitung in einer Grundstromung, Mitt. MPI und AVA, Gottingen, Nr. 35.

Reid, R. C., Prausnitz, J. M. \& Sherwood, T. K. 1977 The Properties of Gases and Liquids, 3rd edn, p. 554. McGraw Hill.

Ricot, F. P. \& Spalding, D. B. 1961 Measurements of the entrainment by axisymmetrical turbulent jets. J. Fluid Mech. 11, 21-32.

Ruden, P. 1933 Turbulente Aurbreitungsvorgänge im Freistrahl, Naturwissenshaften (Jahrg. 21) 21/23, 375-378.

TAYLoR, G. I. 1953 Dispersion of soluble matter in a solvent flowing slowly through a tube. Proc. R. Soc. Lond. A 219, 186-203.

Thring, M. W. \& Newby, M. P. 1953 Combustion length of enclosed turbulent jet flames. Fourth (Intl) Symp. Combustion, pp. 789-796. The William \& Wilkins Co., Baltimore, Maryland.

Townsend, A. A. 1976 The Structure of Turbulent Shear Flow, 2nd edn. Cambridge University Press.

WeddeLL, D. S. 1953 in Hottel, H. C. Burning in laminar and turbulent fuel jets. Fourth (Intl) Symp. Combustion, pp. 97-113. The William \& Wilkins Co., Baltimore, Maryland.

White, F. M. 1974 Viscous Fluid Flow, pp. 505-510. MeGraw-Hill.

WIENER, N. 1949 Extrapolation, Interpolation, and Smoothing of Stationary Time Series. Technology Press (MIT and John Wiley).

Wilson, R. A. M. \& Danckwerts, P. V. 1964 Studies in turbulent mixing - II, A hot air jet. Chem. Engr. Sci. 19, 885-895.

Yakнot, V., She, Z. S. \& Orszag, S. A. 1989 Deviations from the classical Kolmogorov theory of the inertial range of homogeneous turbulence. Phys. Fluids A 1, 289-293. 